\title{
Belief Update within Propositional Fragments
}

\author{
Nadia Creignou \\ Aix Marseille Univ, Université de Toulon, CNRS, \\ LIS, Marseille, France \\ 163 av. de Luminy, 13288, Marseille, France
}

Raïda Ktari

RAIDA.KTARI@ISIMS.USF.TN

Institut Supérieur d'Informatique et de Multimédia de Sfax, Université de Sfax, pôle technologique de Sfax,

Sakiet Ezzit 3021, Tunisie

\section{Odile Papini}

Aix Marseille Univ, Université de Toulon, CNRS,

LIS, Marseille, France

163 av. de Luminy, 13288, Marseille, France

\author{
NADIA.CREIGNOU@UNIV-AMU.FR
}

ODILE.PAPINI@UNIV-AMU.FR

\begin{abstract}
Belief change within the framework of fragments of propositional logic is one of the main and recent challenges in the knowledge representation research area. While previous research works focused on belief revision, belief merging, and belief contraction, the problem of belief update within fragments of classical logic has not been addressed so far. In the context of revision, it has been proposed to refine existing operators so that they operate within propositional fragments, and that the result of revision remains in the fragment under consideration. This approach is not restricted to the Horn fragment but also applicable to other propositional fragments like Krom and affine fragments. We generalize this notion of refinement to any belief change operator. We then focus on a specific belief change operation, namely belief update. We investigate the behavior of the refined update operators with respect to satisfaction of the KM postulates and highlight differences between revision and update in this context.
\end{abstract}

\section{Introduction}

Belief update consists in incorporating into an agent's beliefs new information reflecting a change in her environment. The problem of belief update first appeared in the domain of databases for updating deductive databases (Fagin, Ullman, \& Vardi, 1983). Significant links quickly emerged with works developed in artificial intelligence on belief change, especially on belief revision.

Keller and Winslett (1985), and later Katsuno and Mendelzon (1992) contributed to a better understanding regarding the distinction between belief revision and belief update when they proposed a common framework to represent these operations. Belief revision happens when new information is introduced in a static environment, while belief update occurs in a changing environment. From a logical point of view, when the agent's beliefs are represented by a logical formula, revision makes the models of this formula evolve as a whole towards the closest models of new information. In contrast, update makes each model of this formula locally evolve towards the closest models of new information. 
Postulates characterizing the rational behavior of update operators have been proposed by Katsuno and Mendelzon (KM) (1992) in the same spirit as the seminal AGM postulates for revision (Alchourrón, Gärdenfors, \& Makinson, 1985). Belief update gave rise to several studies, in most cases within the framework of propositional logic, and concrete belief update operators have been proposed mainly according to a semantic (model-based) point of view (Forbus, 1989; del Val \& Shoham, 1994; Dubois \& Prade, 1993; Zhang \& Foo, 2000; Boutilier, 1998; Friedman \& Halpern, 1999; Herzig \& Rifi, 1999; Doherty, Lukaszewicz, \& Madalinska-Bugaj, 2000; Lang, 2007; Delgrande, Jin, \& Pelletier, 2014).

Many studies focused on belief change within the framework of propositional logic fragments, particularly on belief contraction (Booth, Meyer, Varzinczak, \& Wassermann, 2011; Zhuang \& Pagnucco, 2014; Delgrande \& Wassermann, 2013), on belief revision (Cadoli \& Scarcello, 2000; Delgrande \& Peppas, 2015; Zhuang, Pagnucco, \& Zhang, 2013; Putte, 2013; Creignou, Papini, Pichler, \& Woltran, 2014) and more recently on belief merging (Creignou, Papini, Rümmele, \& Woltran, 2016). However, as far as we know, the problem of belief update within fragments of propositional logic has not been addressed so far, except for complexity results in the Horn case (Eiter \& Gottlob, 1992; Liberatore \& Schaerf, 2001). The motivation of such a study is twofold. First, in many applications, the language is restricted a priori. For instance, a rule-based formalization of expert knowledge is much easier to handle for standard users. In the case of update they expect an outcome in the same language. Second, some fragments of propositional logic allow for efficient reasoning methods, and then an outcome of update within such a fragment can be evaluated efficiently. It seems thus natural to investigate how known update operators can be refined such that the result of update remains in the fragment under consideration.

Formally, let $\mathcal{L}^{\prime}$ be a propositional fragment and given two formulas $\psi, \mu \in \mathcal{L}^{\prime}$, the main obstacle hereby is that there is no guarantee that the outcome of an update, denoted by $\psi \diamond \mu$, remains in $\mathcal{L}^{\prime}$ as well. Let us consider the following example inspired from the one used by Katsuno and Mendelzon (1992) where the beliefs describe two objects $A$ and $B$ inside a room. There is a table in the room and the objects may be on the table or not. Suppose $a$ means "object $A$ is on the table" and $b$ means "object $B$ is on the table". Assume that the agent's beliefs are represented by the formula $\psi=a$, which expresses that object $A$ is on the table. Suppose a robot is sent into the room with the instruction to achieve a situation in which either object $A$ or object $B$ is not on the table. This change is represented by the formula $\mu=\neg a \vee \neg b$. The formulas $\psi$ and $\mu$ are Horn formulas, however updating $\psi$ by $\mu$ in using Forbus' (1989) or Winslett's operator (1988) results in a formula equivalent to $\phi=(a \vee b) \wedge(\neg a \vee \neg b)$, which is not a Horn formula and is not equivalent to any Horn formula (because its set of models is not closed under intersection, while this property characterizes the formulas in Horn, 1951) ${ }^{1}$.

In this paper, we generalize the notion of refinement, initially defined for revision (Creignou et al., 2014), to any belief change operator defined from $\mathcal{L} \times \mathcal{L}$ to $\mathcal{L}$ where $\mathcal{L}$ denotes propositional logic. A refinement adapts a belief change operator defined in a propositional setting such that it can be applicable in a propositional fragment. The basic properties of a refinement are first to guarantee the outcome of the belief change operation to remain within the fragment and second to approximate the behavior of the original belief

1. Note that in this example, revision and update do not coincide. 
change operator, in particular to keep the behavior of the original operator unchanged if the result already fits in the fragment. We characterize these refined operators in a constructive way.

We exploit the notion of refinement for belief update operators. We then study how refined belief update operators behave with respect to satisfaction of the KM postulates that characterize rational update operators. Indeed, we show that the basic KM postulates (U1) - (U4) are preserved for any refinement in any fragment. We study the limits of the preservation of the other postulates, as well. For this we focus on the refinements of Forbus' (1989) and Winslett's operators (1988) within the Horn, Krom and affine fragments. Our approach handles a natural extension that consists in investigating update when only the formula representing the initial agent's beliefs, and not necessarily the formula reflecting the new information, is in the fragment. All along this study we shed some light on subtle differences between update and revision.

The paper is organized as follows. We start with some preliminaries. In Section 2.1 we recall some basic facts about propositional logic. In Section 2.2 we define the fragments of propositional logic we are interested in. In Section 2.4 we give a short reminder of belief update. Section 3 deals with refinements in the general context of belief change. In Section 4 we focus on refinements of update operators. Finally we conclude in Section 5.

\section{Preliminaries}

In this section we give the relevant material on propositional logic, characterizable fragments, belief revision and belief update.

\subsection{Propositional Logic}

Let $\mathcal{L}$ be the language of propositional logic built on an infinite countable set of variables (atoms) denoted by $\mathcal{V}$ and equipped with standard connectives $\rightarrow, \vee, \wedge, \neg$, the exclusive or connective $\oplus$, and constants $T, \perp$. A literal is an atom or its negation. A clause is a disjunction of literals. A clause is called Horn if at most one of its literals is positive; Krom if it consists of at most two literals. A $\oplus$-clause is defined like a clause but using exclusive - instead of standard - disjunction.

We identify $\mathcal{L}_{\text {Horn }}\left(\right.$ resp., $\mathcal{L}_{\text {Krom }}, \mathcal{L}_{\text {affine }}$ ) as the set of all formulas in $\mathcal{L}$ being conjunctions of Horn clauses (resp., Krom clauses, $\oplus$-clauses).

Let $\mathcal{U}$ be a finite set of atoms. An interpretation over $\mathcal{U}$ is represented either by a set $m \subseteq \mathcal{U}$ of atoms (corresponding to the variables set to true) or by its corresponding characteristic bit-vector of length $|\mathcal{U}|$, the atoms being considered in lexicographical order. For instance if we consider $\mathcal{U}=\left\{x_{1}, \ldots, x_{6}\right\}$, the interpretation $x_{1}=x_{3}=x_{6}=1$ and $x_{2}=x_{4}=x_{5}=0$ will be represented either by $\left\{x_{1}, x_{3}, x_{6}\right\}$ or by $(1,0,1,0,0,1)$.

For any formula $\phi$, let $\operatorname{Var}(\phi)$ denote the set of variables occurring in $\phi$. As usual, if an interpretation $m$ defined over $\mathcal{U}$ satisfies a formula $\phi$ such that $\operatorname{Var}(\phi) \subseteq \mathcal{U}$, we call $m$ a model of $\phi . \operatorname{By} \operatorname{Mod}(\phi)$ we denote the set of all models (over $\mathcal{U}$ ) of $\phi$.

A formula $\psi$ is complete over $\mathcal{U}$ if $\operatorname{Var}(\psi) \subseteq \mathcal{U}$ and if for any $\mu \in \mathcal{L}$ such that $\operatorname{Var}(\mu) \subseteq \mathcal{U}$, we have $\psi \models \mu$ or $\psi \models \neg \mu$. In an equivalent way, a satisfiable formula $\psi$ is complete over 
$\mathcal{U}^{2}$ if it has exactly one model over $\mathcal{U}$. Moreover, $\psi \models \phi$ if $\operatorname{Mod}(\psi) \subseteq \operatorname{Mod}(\phi)$ and $\psi \equiv \phi$ if $\operatorname{Mod}(\psi)=\operatorname{Mod}(\phi)$. For fragments $\mathcal{L}^{\prime} \subseteq \mathcal{L}$, we use $T_{\mathcal{L}^{\prime}}(\psi)=\left\{\phi \in \mathcal{L}^{\prime} \mid \psi \models \phi\right\}$.

\subsection{Characterizable Fragments of Propositional Logic}

Let $\mathcal{B}$ be the set of Boolean functions $\beta:\{0,1\}^{k} \rightarrow\{0,1\}$ with $k \geqslant 1$, that are symmetric (i.e., for all permutations $\left.\sigma, \beta\left(x_{1}, \ldots, x_{k}\right)=\beta\left(x_{\sigma(1)}, \ldots, x_{\sigma(k)}\right)\right)$, and 0 - and 1-reproductive (i.e., for every $x \in\{0,1\}, \beta(x, \ldots, x)=x)$. Examples of such functions are: The binary AND function denoted by $\wedge$, the ternary MAJORITY function, $\operatorname{maj}_{3}(x, y, z)=1$ if at least two of the variables $x, y$ and $z$ are set to 1 , and the ternary XOR function $\oplus_{3}(x, y, z)=x \oplus y \oplus z$.

Recall that we consider interpretations also as bit-vectors. We thus extend Boolean functions to interpretations by applying coordinate-wise the original function. So, if $m_{1}, \ldots, m_{k} \in$ $\{0,1\}^{n}$, then $\beta\left(m_{1}, \ldots, m_{k}\right)$ is defined by

$$
\left(\beta\left(m_{1}[1], \ldots, m_{k}[1]\right), \ldots, \beta\left(m_{1}[n], \ldots, m_{k}[n]\right)\right),
$$

where $m[i]$ is the $i$-th coordinate of the interpretation $m$. The next definition gives a general formal definition of closure.

Definition 1. Given a set $\mathcal{M} \subseteq 2^{\mathcal{U}}$ of interpretations and $\beta \in \mathcal{B}$, we define $C l_{\beta}(\mathcal{M})$, the closure of $\mathcal{M}$ under $\beta$, as the smallest set of interpretations that contains $\mathcal{M}$ and that is closed under $\beta$, i.e., if $m_{1}, \ldots, m_{k} \in C l_{\beta}(\mathcal{M})$, then $\beta\left(m_{1}, \ldots, m_{k}\right) \in C l_{\beta}(\mathcal{M})$.

For instance it is well-known that the set of models of any Horn formula is closed under $\wedge$, and actually this property characterizes Horn formulas.

Closures satisfy monotonicity: if $\mathcal{M} \subseteq \mathcal{N}$, then $C l_{\beta}(\mathcal{M}) \subseteq C l_{\beta}(\mathcal{N})$. Moreover, if $|\mathcal{M}|=1$, then $C l_{\beta}(\mathcal{M})=\mathcal{M}$ (because by assumption $\beta$ is 0 - and 1-reproducing); finally, we always have $C l_{\beta}(\varnothing)=\varnothing$.

We can now use these concepts to identify fragments of propositional logic. Additionally, we want fragments to fulfill some natural properties and for technical reasons we require closure under conjunction.

Definition 2. Let $\beta \in \mathcal{B}$. A set $\mathcal{L}^{\prime} \subseteq \mathcal{L}$ of propositional formulas is a $\beta$-fragment (or a characterizable fragment) if: (i) For all $\psi \in \mathcal{L}^{\prime}, \operatorname{Mod}(\psi)=C l_{\beta}(\operatorname{Mod}(\psi))$. (ii) For all $\mathcal{M} \subseteq 2^{\mathcal{U}}$ with $\mathcal{M}=C l_{\beta}(\mathcal{M})$ there exists $\psi \in \mathcal{L}^{\prime}$ with $\operatorname{Mod}(\psi)=\mathcal{M}$. (iii) If $\phi, \psi \in \mathcal{L}^{\prime}$ then $\phi \wedge \psi \in \mathcal{L}^{\prime}$.

We will often (implicitly) use the following fact: Let $\mu$ be a formula in $\mathcal{L}$ and $\mathcal{L}^{\prime}$ be a $\beta$-fragment. Let $\tilde{\mu}$ be a formula in $\mathcal{L}^{\prime}$ such that $\operatorname{Mod}(\tilde{\mu})=C l_{\beta}(\operatorname{Mod}(\mu))$ (such a formula exists according to (ii) in Definition 2). Then $T_{\mathcal{L}^{\prime}}(\mu)=T_{\mathcal{L}^{\prime}}(\tilde{\mu})$.

Many fragments of propositional logic allow for efficient reasoning methods. When representing knowledge, storing beliefs as a formula of a known tractable class is thus of interest. The most famous characterizable fragments, which are the largest in which satisfiability is tractable, are: $\mathcal{L}_{\text {Horn }}$ which is an $\wedge$-fragment, $\mathcal{L}_{\text {Krom }}$ which is a maj $_{3}$-fragment and $\mathcal{L}_{\text {affine }}$ which is a $\oplus_{3}$-fragment (Horn, 1951; Schaefer, 1978).

2. When $\mathcal{U}$ is not mentioned, it implicitly means that $\mathcal{U}$ is the set of variables occurring in formulas under consideration 
An immediate generalization of our framework to fragments characterized by a closure property under a finite number of functions (and not only one), leads to infinitely many fragments, which are organized in a lattice, known as Post's (1941) lattice. The complexity of many computational tasks has been studied in these fragments (see the survey in Creignou \& Vollmer, 2008). The complexity of reasoning tasks within the Krom fragment has been recently investigated (Creignou, Pichler, \& Woltran, 2017).

\subsection{Belief Revision}

Belief revision consists in incorporating a new belief, changing as few as possible of the original beliefs while preserving consistency. More formally, a revision operator denoted by $\circ$, is a function from $\mathcal{L} \times \mathcal{L}$ to $\mathcal{L}$ that maps two formulas $\psi$ (the initial agent's beliefs) and $\mu$ (new information) to a new formula $\psi \circ \mu$ (the revised agent's beliefs).

In the AGM paradigm (Alchourrón et al., 1985), postulates were proposed for belief revision when beliefs are modeled by a theory (or belief set), Katsuno and Mendelzon (1991) reformulated them when a theory is represented by a propositional formula. We recall the KM postulates for belief revision.

Let $\psi, \psi_{1}, \psi_{2}, \mu, \mu_{1}, \mu_{2} \in \mathcal{L}$.

(R1) $\psi \circ \mu \models \mu$.

(R2) If $\psi \wedge \mu$ is satisfiable, then $\psi \circ \mu \equiv \psi \wedge \mu$.

(R3) If $\mu$ is satisfiable, then so is $\psi \circ \mu$.

(R4) If $\psi_{1} \equiv \psi_{2}$ and $\mu_{1} \equiv \mu_{2}$, then $\psi_{1} \circ \mu_{1} \equiv \psi_{2} \circ \mu_{2}$.

(R5) $(\psi \circ \mu) \wedge \phi \models \psi \circ(\mu \wedge \phi)$.

(R6) If $(\psi \circ \mu) \wedge \phi$ is satisfiable, then also $\psi \circ(\mu \wedge \phi) \models(\psi \circ \mu) \wedge \phi$.

The meaning of these postulates is the following. Postulate (R1) specifies that the added formula belongs to the revised belief set. Postulate (R2) is concerned with following issue: if the added formula does not contradict the initial belief set then the revised belief set is represented by the conjunction of the added formula and the formula representing the initial belief set, in other words if the incorporation of new information does not cause problem, we just add the new belief to the existing knowledge. Postulate (R3) ensures that no inconsistency is introduced in the revised belief set. Postulate (R4) expresses the principle of irrelevance of the syntax, and (R5) and (R6) state that revising by the conjunction of two pieces of information amounts to a revision by the first one and a conjunction of the second one whenever possible (whenever the second piece of information does not contradict any belief resulting from the first revision).

Katsuno and Mendelzon (1991) showed that a revision satisfying the AGM postulates is equivalent to a total preorder on interpretations, which reflects a plausibility ordering on interpretations. More formally, a faithful assignment is a function that maps any propositional formula $\psi$ to a pre-order over interpretations $\leqslant \psi$ such that:

1) If $\omega \models \psi$ and $\omega^{\prime} \models \varphi$, then $\omega={ }_{\psi} \omega^{\prime}$.

2) If $\omega \models \psi$ and $\omega^{\prime} \models \psi$, then $\omega<_{\psi} \omega^{\prime}$.

3) If $\psi_{1} \equiv \psi_{2}$ then $\leqslant_{1}=\leqslant \psi_{2}$.

They provided the following representation theorem. 
Theorem 3. (Katsuno \& Mendelzon, 1991) A revision operator $\circ$ satisfies the postulates (R1)-(R6) if and only if there exists a faithful assignment that maps each formula $\psi$ to a total preorder $\leqslant_{\psi}$ such that $\operatorname{Mod}(\psi \circ \mu)=\operatorname{Min}\left(\operatorname{Mod}(\mu), \leqslant_{\psi}\right)$.

\subsection{Belief Update}

Belief update consists in incorporating into an agent's beliefs new information reflecting a change in her environment. More formally, an update operator, denoted by $\diamond$, is a function from $\mathcal{L} \times \mathcal{L}$ to $\mathcal{L}$ that maps two formulas $\psi$ (the initial agent's beliefs) and $\mu$ (new information) to a new formula $\psi \diamond \mu$ (the updated agent's beliefs). We recall the KM postulates for belief update (Katsuno \& Mendelzon, 1991).

Let $\psi, \psi_{1}, \psi_{2}, \mu, \mu_{1}, \mu_{2} \in \mathcal{L}$.

(U1) $\psi \diamond \mu \models \mu$.

(U2) If $\psi \models \mu$, then $\psi \diamond \mu \equiv \psi$.

(U3) If $\psi$ and $\mu$ are satisfiable then so is $\psi \diamond \mu$.

(U4) If $\psi_{1} \equiv \psi_{2}$ and $\mu_{1} \equiv \mu_{2}$, then $\psi_{1} \diamond \mu_{1} \equiv \psi_{2} \diamond \mu_{2}$.

(U5) $\quad(\psi \diamond \mu) \wedge \phi=\psi \diamond(\mu \wedge \phi)$.

(U6) If $\left(\psi \diamond \mu_{1}\right) \models \mu_{2}$ and $\left(\psi \diamond \mu_{2}\right) \models \mu_{1}$, then $\psi \diamond \mu_{1} \equiv \psi \diamond \mu_{2}$.

(U7) If $\psi$ is complete, then $\left(\psi \diamond \mu_{1}\right) \wedge\left(\psi \diamond \mu_{2}\right) \models \psi \diamond\left(\mu_{1} \vee \mu_{2}\right)$.

(U8) $\quad\left(\psi_{1} \vee \psi_{2}\right) \diamond \mu \equiv\left(\psi_{1} \diamond \mu\right) \vee\left(\psi_{2} \diamond \mu\right)$.

(U9) If $\psi$ is complete and $(\psi \diamond \mu) \wedge \phi$ is satisfiable, then $\psi \diamond(\mu \wedge \phi) \models(\psi \diamond \mu) \wedge \phi$.

These postulates have been discussed in several papers (e.g., Herzig \& Rifi, 1999). Postulate (U1) says that the models of the updated agent's beliefs have to be models of new information. Postulate (U4) states the irrelevance of syntax. Postulate (U5) expresses minimality of change. The three postulates (U1), (U4) and (U5) directly correspond to the belief revision postulates (R1), (R4) and (R5) respectively. Postulate (U2) differs from (R2), the latter stating that if $\psi \wedge \mu$ is satisfiable then $\psi \circ \mu \equiv \psi \wedge \mu$. A consequence of (U2) for update is that once an inconsistency is introduced in the initial beliefs there is no way to eliminate it (Katsuno \& Mendelzon, 1991). Note that this is not the case for belief revision. Furthermore, (U3) is a weaker version of (R3). The latter states that if $\mu$ is satisfiable then so is $\psi \circ \mu$, while in order to ensure the consistency of the result of update (U3) requires an additional condition, namely that the initial beliefs be consistent as well. Postulates (U6), (U7) and (U8) are specific to update operators. The eighth postulate (U8), which means that an update operator should give each of the models of the initial beliefs equal consideration, is considered as the most "uncontroversial" one. Finally, (U9) is a weaker version of (R6), it is similar but restricted to complete formulas $\psi$.

Katsuno and Mendelzon (1991)showed that an update operator corresponds to a set of preorders on interpretations. More formally, a pointwise faithful assignment is a function that maps any interpretation $m$ to a pre-order over interpretations $\leqslant_{m}$, such that for any interpretation $m^{\prime}$, if $m^{\prime} \neq m$ then $m<_{m} m^{\prime}$. They provided the following representation theorem.

Theorem 4. (Katsuno 83 Mendelzon, 1992) 
- An update operator $\diamond$ satisfies the postulates (U1)-(U9) if and only if there exists a pointwise faithful assignment that maps each interpretation $m$ to a total preorder $\leqslant_{m}$ such that $\operatorname{Mod}(\psi \diamond \mu)=\bigcup_{m \in \operatorname{Mod}(\psi)} \min \left(\operatorname{Mod}(\mu), \leqslant_{m}\right)$.

- An update operator $\diamond$ satisfies the postulates (U1)-(U8) if and only if there exists a pointwise faithful assignment that maps each interpretation $m$ to a partial preorder $\leqslant_{m}$ such that $\operatorname{Mod}(\psi \diamond \mu)=\bigcup_{m \in \operatorname{Mod}(\psi)} \min \left(\operatorname{Mod}(\mu), \leqslant_{m}\right)$.

The representation theorems, Theorem 3 and Theorem 4, pinpoint the differences between revision and update. Update stems from a pointwise minimization, model by model of $\psi$, while revision stems from a global minimization on all the models of $\psi$. Update operators, for each model $m$ of $\psi$, select the set of models of $\mu$ that are the closest to $m$, while revision operators select the set of models of $\mu$ that are the closest to the set of models of $\psi$. Note that when there exists only one model of $\psi$ (which is the case when $\psi$ is complete) revision and update coincide.

The following example illustrates the difference between revision and update.

Example 5. We come back to the example given in the introduction where the beliefs describe two objects $A$ and $B$ inside a room. The agent's beliefs are represented by the formula $\psi=a$, which expresses that object $A$ is on the table. Let us recall that a robot is sent into the room with the instruction to achieve a situation in which either object $A$ or object $B$ is not on the table. This change is represented by the formula $\mu=\neg a \vee \neg b$. We have $\psi, \mu \in \mathcal{L}$ with $\operatorname{Mod}(\psi)=\{\{a\},\{a, b\}\}$ and $\operatorname{Mod}(\mu)=\{\{a\},\{b\}, \varnothing\}$. Let $m, m^{\prime}$ be two interpretations, $m \Delta m^{\prime}$ denotes the symmetric difference between $m$ and $m^{\prime}$. The global minimization of the cardinality of the symmetric difference between the models of $\psi$ and the models of $\mu$ provides $\operatorname{Mod}(\psi \circ \mu)=\{\{a\}\}$. In contrast, the minimization of the cardinality of the symmetric difference between each model of $\psi$ and the models of $\mu$ gives $\operatorname{Mod}(\psi \diamond \mu)=\{\{a\},\{b\}\}$. Note that revision selects $\{a\}$ as the only model of the changed beliefs. However after the robot's action, all we know is that either object $A$ or object $B$ is not on the table. There is no reason to conclude that only object $B$ is not on the table as does revision, which excludes the situation where object $A$ is not on the table.

Several update operators have been proposed. We now recall the two best known modelbased update operators on which we will focus, namely Forbus' (1989) and Winslett's operators (1988). In these model-based update operators the closeness between models relies on the symmetric difference between models, that is the set of propositional variables on which they differ.

Forbus' (1989) operator was introduced in the context of qualitative physics. This operator is analogous to Dalal's (1988) revision operator and measures minimality of change by cardinality of model change. More formally, let $\psi$ and $\mu$ be two propositional formulas, and $m$ and $m^{\prime}$ be two interpretations, $m \Delta m^{\prime}$ denotes the symmetric difference between $m$ and $m^{\prime}$ and $|\Delta|_{m}^{\min }(\mu)$ denotes the minimum number of variables in which $m$ and a model of $\mu$ differ and is defined as $\min \left\{\left|m \Delta m^{\prime}\right|: m^{\prime} \in \operatorname{Mod}(\mu)\right\}$. Forbus' operator is now defined as: $\operatorname{Mod}\left(\psi \diamond_{F} \mu\right)=\bigcup_{m \in \operatorname{Mod}(\psi)}\left\{m^{\prime} \in \operatorname{Mod}(\mu):\left|m \Delta m^{\prime}\right|=|\Delta|_{m}^{\min }(\mu)\right\}$. This operator satisfies (U1)-(U8) (Katsuno \& Mendelzon, 1991) and (U9) (Herzig \& Rifi, 1999). This update operator is illustrated in the following example. 


\begin{tabular}{l|l|l|l|l|l|l|l}
$\operatorname{Mod}(\psi)$ & \multicolumn{9}{|c}{$\operatorname{Mod}(\mu)$} \\
\hline & $\{\mathrm{b}, \mathrm{c}\}$ & $\{\mathrm{c}, \mathrm{d}\}$ & $\{\mathrm{a}, \mathrm{b}, \mathrm{d}\}$ & $\{\mathrm{c}\}$ & $\{\mathrm{d}\}$ & $\{\mathrm{b}\}$ & $\varnothing$ \\
\hline$\{\mathrm{a}, \mathrm{b}, \mathrm{c}\}$ & $\mathbf{1}$ & 3 & 2 & 2 & 4 & 2 & 3 \\
\hline$\{\mathrm{a}, \mathrm{b}, \mathrm{c}, \mathrm{d}, \mathrm{e}\}$ & 3 & 3 & $\mathbf{2}$ & 4 & 4 & 4 & 5 \\
\hline
\end{tabular}

Table 1: Example for $\diamond_{F}$

\begin{tabular}{l|l|l|l|l|l|l|l}
$\operatorname{Mod}(\psi)$ & \multicolumn{7}{c}{$\operatorname{Mod}(\mu)$} \\
\hline & $\{\mathrm{b}, \mathrm{c}\}$ & $\{\mathrm{c}, \mathrm{d}\}$ & $\{\mathrm{a}, \mathrm{b}, \mathrm{d}\}$ & $\{\mathrm{c}\}$ & $\{\mathrm{d}\}$ & $\{\mathrm{b}\}$ & $\varnothing$ \\
\hline$\{\mathrm{a}, \mathrm{b}, \mathrm{c}\}$ & $\{\mathbf{a}\}$ & $\{\mathrm{a}, \mathrm{b}, \mathrm{d}\}$ & $\{\mathbf{c}, \mathbf{d}\}$ & $\{\mathrm{a}, \mathrm{b}\}$ & $\{\mathrm{a}, \mathrm{b}, \mathrm{c}, \mathrm{d}\}$ & $\{\mathrm{a}, \mathrm{c}\}$ & $\{\mathrm{a}, \mathrm{b}, \mathrm{c}\}$ \\
\hline$\{\mathrm{a}, \mathrm{b}, \mathrm{c}, \mathrm{d}, \mathrm{e}\}$ & $\{\mathbf{a}, \mathbf{d}, \mathbf{e}\}$ & $\{\mathbf{a}, \mathbf{b}, \mathbf{e}\}$ & $\{\mathbf{c}, \mathbf{e}\}$ & $\{\mathrm{a}, \mathrm{b}, \mathrm{d}, \mathrm{e}\}$ & $\{\mathrm{a}, \mathrm{b}, \mathrm{c}, \mathrm{e}\}$ & $\{\mathrm{a}, \mathrm{c}, \mathrm{d}, \mathrm{e}\}$ & $\{\mathrm{a}, \mathrm{b}, \mathrm{c}, \mathrm{d}, \mathrm{e}\}$ \\
\hline
\end{tabular}

Table 2: Example for $\diamond_{W}$

Example 6. Let $\psi, \mu \in \mathcal{L}$ such that $\operatorname{Mod}(\psi)=\{\{a, b, c\},\{a, b, c, d, e\}\}$ and $\operatorname{Mod}(\mu)=$ $\{\{b, c\},\{c, d\},\{a, b, d\},\{c\},\{d\},\{b\}, \varnothing\}$. The result of update could be read in Table 1. Each line of the table gives the cardinalities of the symmetric differences between the corresponding model of $\psi$ and the models of $\mu$. The minimal cardinalities are written in bold. Hence $\operatorname{Mod}\left(\psi \diamond_{F} \mu\right)=\{\{b, c\},\{a, b, d\}\}$.

Winslett's (1988) operator, also called PMA (Possible Models Approach) was introduced for reasoning about actions and change. This operator is analogous to Satoh's (1988) revision operator and interprets minimal change in terms of set inclusion instead of cardinality on model difference. More formally, $\Delta_{m}^{\min }(\mu)$ denotes the minimal difference between $m$ and a model of $\mu$ and is defined as $\min _{\subseteq}\left(\left\{m \Delta m^{\prime}: m^{\prime} \in \operatorname{Mod}(\mu)\right\}\right)$. Winslett's operator is now defined as: $\operatorname{Mod}\left(\psi \diamond_{W} \mu\right)=\bigcup_{m \in \operatorname{Mod}(\psi)}\left\{m^{\prime} \in \operatorname{Mod}(\mu): m \Delta m^{\prime} \in \Delta_{m}^{\min }(\mu)\right\}$. This operator satisfies (U1) - (U8) (Katsuno \& Mendelzon, 1991) but does not satisfy (U9) (Ktari, 2016). Winslett's operator $\diamond_{W}$ behaves differently from Forbus' operator $\diamond_{F}$ (1989) as illustrated in the following example.

Example 7. Let $\psi, \mu \in \mathcal{L}$ from Example 6. The result of update could be read in Table 2. Each line of the table gives the symmetric differences between the corresponding model of $\psi$ and the models of $\mu$. The minimal subsets with respect to set inclusion are written in bold. Hence $\operatorname{Mod}\left(\psi \diamond_{W} \mu\right)=\{\{b, c\},\{c, d\},\{a, b, d\}\} \neq \operatorname{Mod}\left(\psi \diamond_{F} \mu\right)$.

In this paper, we are interested in update operators which are tailored for certain fragments. We say that $\diamond$ satisfies the postulates $(\mathrm{U} i)(i \in\{1, \ldots, 9\})$ in a fragment $\mathcal{L}^{\prime} \subseteq \mathcal{L}$ if these postulates hold when restricted to formulas from $\mathcal{L}^{\prime}$.

\section{Refinements of Belief Change Operators}

Refinements have been defined within the context of belief revision (Creignou et al., 2014) and may be naturally considered for any belief change operation.

The idea is to use well-established belief change operators in order to define rational belief change operators that are well-suited for characterizable fragments of propositional 
logic. Given a propositional fragment $\mathcal{L}^{\prime}$ and a propositional belief change operator $\triangle$, a refinement of $\triangle$ consists of a new operator $\boldsymbol{\Delta}$, which is built from $\triangle$ and not too different from $\triangle$, that operates within $\mathcal{L}^{\prime}$ and is such that the result of change remains in $\mathcal{L}^{\prime}$. Roughly speaking the goal is that the difference of behavior between $\triangle$ and $\boldsymbol{\Delta}$ obeys a kind of principle of minimal change in the sense that if the original operator $\triangle$ gives a result that is already in the fragment, then the refined operator should do nothing more, and in any case it should not increase the logical consequences of the original result. In the following we first define formally a few natural basic properties for refinements, then we show how such refinements can be explicitly obtained.

Definition 8. Let $\mathcal{L}^{\prime}$ be a propositional fragment and $\triangle: \mathcal{L} \times \mathcal{L} \rightarrow \mathcal{L}$ a belief change operator. We call an operator $\boldsymbol{\Delta}: \mathcal{L}^{\prime} \times \mathcal{L}^{\prime} \rightarrow \mathcal{L}^{\prime}$ a $\triangle$-refinement for $\mathcal{L}^{\prime}$ if it satisfies the following properties, for each $\psi, \psi^{\prime}, \mu, \mu^{\prime} \in \mathcal{L}^{\prime}$.

- consistency: $\psi \mathbf{\Delta} \mu$ is satisfiable if and only if $\psi \Delta \mu$ is satisfiable.

- equivalence: If $\psi \Delta \mu \equiv \psi^{\prime} \triangle \mu^{\prime}$ then, $\psi \boldsymbol{\Delta} \mu \equiv \psi^{\prime} \boldsymbol{\Delta} \mu^{\prime}$.

- containment: $T_{\mathcal{L}^{\prime}}(\psi \triangle \mu) \subseteq T_{\mathcal{L}^{\prime}}(\psi \boldsymbol{\Lambda} \mu)$.

- invariance: If $\psi \triangle \mu \in \mathcal{L}^{\prime}$, then $T_{\mathcal{L}^{\prime}}(\psi \Delta \mu)=T_{\mathcal{L}^{\prime}}(\psi \triangle \mu)$.

Let us briefly discuss these properties. The first two conditions are rather independent from $\mathcal{L}^{\prime}$, but relate the refined operator $\boldsymbol{\Delta}$ to the original belief change $\Delta$ in certain ways. To be more precise, consistency states that the refined operator $\boldsymbol{\Delta}$ should yield a consistent belief change exactly if the original operator $\triangle$ does so. Equivalence means that the definition of the $\boldsymbol{\Lambda}$-operator should not be syntax-dependent, belief changes which are equivalent w.r.t $\triangle$ are also equivalent w.r.t. $\boldsymbol{\Delta}$. Containment ensures that $\boldsymbol{\Delta}$ can be seen as a form of approximation of $\triangle$ when applied in the $\mathcal{L}^{\prime}$ fragment, while invariance states that in case $\triangle$ behaves as expected (i.e., the belief change is contained in $\mathcal{L}^{\prime}$ ) there is no need for $\boldsymbol{\Delta}$ to do something additional.

When considering a model-based operator $\triangle$ it seems that such a refinement can be obtained as follows. Let $\mathcal{L}^{\prime}$ be a $\beta$-fragment and $\psi$ and $\mu$ two formulas in $\mathcal{L}^{\prime}$. Let $\triangle$ be a model-based belief change operator. In order to set a refinement, we first compute the set of models of $\psi \triangle \mu$, denoted by $\mathcal{M}$, we then apply a mapping to $\mathcal{M}$ in order to obtain a set of models $\mathcal{N}$ that is a set of models of a formula in $\mathcal{L}^{\prime}$. We call such a mapping a $\beta$-mapping since $\mathcal{N}$ has to be closed under $\beta$. In the following we prove that indeed all possible refinements can be obtained that way.

This characterization of all possible refinements requires the definition of the notion of $\beta$-mapping.

Definition 9. Given $\beta \in \mathcal{B}$, we define a $\beta$-mapping, $f_{\beta}$, as an application from sets of models into sets of models, $f_{\beta}: 2^{2^{\mathcal{U}}} \longrightarrow 2^{2^{\mathcal{U}}}$, such that for every $\mathcal{M} \subseteq 2^{\mathcal{U}}$ :

1. $C l_{\beta}\left(f_{\beta}(\mathcal{M})\right)=f_{\beta}(\mathcal{M})$, i.e., $f_{\beta}(\mathcal{M})$ is closed under $\beta$.

2. $f_{\beta}(\mathcal{M}) \subseteq C l_{\beta}(\mathcal{M})$. 
3. if $\mathcal{M}=C l_{\beta}(\mathcal{M})$, then $f_{\beta}(\mathcal{M})=\mathcal{M}$.

4. If $\mathcal{M} \neq \varnothing$, then $f_{\beta}(\mathcal{M}) \neq \varnothing$.

As explained above the underlying idea of functions $f_{\beta}$ is to take a set of models $\operatorname{Mod}(\psi \triangle \mu)$ and to return a set of models $f_{\beta}(\operatorname{Mod}(\psi \triangle \mu))$, thus defining a refinement of the operator $\triangle$. The outcome has to be closed under $\beta$ (1) since we want to get a belief change into formulas from the $\beta$-fragment, and should not add any further interpretations (2) in order to satisfy containment, cf. Definition 8. Since we want to capture refinements of operators there is no need to change the behavior of the original operator as long as it provides a result in the desired fragment (3). Property (4) takes care of consistency, cf. Definition 8.

Thus, the concept of $\beta$-mapping allows us to define a family of refined operators for the fragments of propositional logic as follows.

Definition 10. Let $\triangle: \mathcal{L} \times \mathcal{L} \longrightarrow \mathcal{L}$ be a belief change operator and $\mathcal{L}^{\prime} \subseteq \mathcal{L}$ be a $\beta$-fragment of propositional logic with $\beta \in \mathcal{B}$. For a $\beta$-mapping $f_{\beta}$, we denote with $\triangle^{f_{\beta}}: \mathcal{L}^{\prime} \times \mathcal{L}^{\prime} \longrightarrow \mathcal{L}^{\prime}$ the belief change operator for $\mathcal{L}^{\prime}$ defined as $\operatorname{Mod}\left(\psi \triangle^{f_{\beta}} \mu\right):=f_{\beta}(\operatorname{Mod}(\psi \triangle \mu))$. The class $\left[\triangle, \mathcal{L}^{\prime}\right]$ contains all operators $\triangle^{f_{\beta}}$ where $f_{\beta}$ is a $\beta$-mapping.

The next proposition is central in reflecting that the above class captures all refined operators we had in mind. A similar result was obtained in Creignou et al. (2014) for basic (revision) operators, i.e., operators satisfying $\top \triangle \mu \equiv \mu$. This assumption was used to prove that any $\triangle$-refinement can be defined through a $\beta$-mapping. We give here an alternative proof that does not rely on this assumption.

Proposition 11. Let $\triangle: \mathcal{L} \times \mathcal{L} \longrightarrow \mathcal{L}$ be a belief change operator and $\mathcal{L}^{\prime} \subseteq \mathcal{L}$ be a characterizable fragment of propositional logic. Then, $\left[\triangle, \mathcal{L}^{\prime}\right]$ is the set of all $\triangle$-refinements for $\mathcal{L}^{\prime}$.

Proof. Since $\mathcal{L}^{\prime}$ is a characterizable fragment it is also a $\beta$-fragment for some $\beta \in \mathcal{B}$. We first show that any operator from the class $\left[\triangle, \mathcal{L}^{\prime}\right]$ is a $\triangle$-refinement of $\mathcal{L}^{\prime}$.

Let $\triangle^{f_{\beta}} \in\left[\triangle, \mathcal{L}^{\prime}\right]$. We have to show that it satisfies the properties of Definition 8. Consistency for $\triangle^{f_{\beta}}$ : Let $\psi, \mu \in \mathcal{L}^{\prime}$. If $\operatorname{Mod}(\psi \triangle \mu) \neq \varnothing$ then $\operatorname{Mod}\left(\psi \triangle^{f_{\beta}} \mu\right)=f_{\beta}(\operatorname{Mod}(\psi \triangle \mu)) \neq$ $\varnothing$ by Property 4 in Definition 9 . In case, $\operatorname{Mod}(\psi \triangle \mu)=\varnothing$, we make use of the fact that $C l_{\beta}(\varnothing)=\varnothing$ holds for all $\beta \in \mathcal{B}$. By Property 2 in Definition 9 , we get $\operatorname{Mod}\left(\psi \triangle^{f_{\beta}} \mu\right)=$ $f_{\beta}(\operatorname{Mod}(\psi \triangle \mu)) \subseteq C l_{\beta}(\operatorname{Mod}(\psi \triangle \mu))=\varnothing$.

Equivalence for $\triangle^{f_{\beta}}$ is clear by definition and since $f_{\beta}$ is defined on sets of models.

To show containment for $\triangle^{f_{\beta}}$, let $\phi \in T_{\mathcal{L}^{\prime}}(\psi \triangle \mu)$, i.e., $\phi \in \mathcal{L}^{\prime}$ and $\operatorname{Mod}(\psi \triangle \mu) \subseteq \operatorname{Mod}(\phi)$. We have $C l_{\beta}(\operatorname{Mod}(\psi \triangle \mu)) \subseteq C l_{\beta}(\operatorname{Mod}(\phi))$ by monotonicity of $C l_{\beta}$. By Property 2 of Definition $9, \operatorname{Mod}\left(\psi \triangle^{f_{\beta}} \mu\right) \subseteq C l_{\beta}(\operatorname{Mod}(\psi \triangle \mu))$. Since $\phi \in \mathcal{L}^{\prime}$ we have $C l_{\beta}(\operatorname{Mod}(\phi))=$ $\operatorname{Mod}(\phi)$. Thus, $\operatorname{Mod}\left(\psi \triangle^{f_{\beta}} \mu\right) \subseteq \operatorname{Mod}(\phi)$, i.e., $\phi \in T_{\mathcal{L}^{\prime}}\left(\psi \triangle^{f_{\beta}} \mu\right)$.

Finally, we require invariance for $\triangle^{f_{\beta}}$ : In case $\psi \triangle \mu \in \mathcal{L}^{\prime}$, we have $C l_{\beta}(\operatorname{Mod}(\psi \triangle \mu))=$ $\operatorname{Mod}(\psi \triangle \mu)$ since $\mathcal{L}^{\prime}$ is a $\beta$-fragment. By Property 3 in Definition 9 , we have $\operatorname{Mod}\left(\psi \triangle^{f_{\beta}} \mu\right)=$ $f_{\beta}(\operatorname{Mod}(\psi \triangle \mu))=\operatorname{Mod}(\psi \triangle \mu)$. Thus $T_{\mathcal{L}^{\prime}}\left(\psi \triangle^{f_{\beta}} \mu\right)=T_{\mathcal{L}^{\prime}}(\psi \triangle \mu)$ as required.

For the converse, let $\boldsymbol{\Delta}$ be a $\triangle$-refinement for $\mathcal{L}^{\prime}$. We show that $\boldsymbol{\Delta} \in\left[\triangle, \mathcal{L}^{\prime}\right]$. Let $f$ be defined as follows for any set $\mathcal{M}$ of interpretations: $f(\varnothing)=\varnothing$ and for $\mathcal{M} \neq \varnothing$, if there 
exists a pair $\left(\psi_{\mathcal{M}}, \mu_{\mathcal{M}}\right)$ of formulas from $\mathcal{L}^{\prime}$ such that $\operatorname{Mod}\left(\psi_{\mathcal{M}} \triangle \mu_{\mathcal{M}}\right)=\mathcal{M}$, then we define $f(\mathcal{M})=\operatorname{Mod}\left(\psi_{\mathcal{M}} \boldsymbol{\Delta} \mu_{\mathcal{M}}\right)$, otherwise $f(\mathcal{M})=C l_{\beta}(\mathcal{M})$. Thus the refined operator $\boldsymbol{\Delta}$ behaves like the operator $\triangle^{f}$.

We show that such a mapping $f$ is a $\beta$-mapping. Note that since $\boldsymbol{\Delta}$ is a $\beta$-refinement, it satisfies the property of equivalence, thus the actual choice of the pair $\left(\psi_{\mathcal{M}}, \mu_{\mathcal{M}}\right)$ is not relevant, i.e., given $\mathcal{M}$, and pairs $\left(\psi_{\mathcal{M}}, \mu_{\mathcal{M}}\right),\left(\psi_{\mathcal{M}}^{\prime}, \mu_{\mathcal{M}}^{\prime}\right)$ such that $\operatorname{Mod}\left(\psi_{\mathcal{M}} \triangle \mu_{\mathcal{M}}\right)=$ $\operatorname{Mod}\left(\psi_{\mathcal{M}}^{\prime} \triangle \mu_{\mathcal{M}}^{\prime}\right)=\mathcal{M}$, we have that $\psi_{\mathcal{M}} \mathbf{\Delta} \mu_{\mathcal{M}}$ is equivalent to $\psi_{\mathcal{M}}^{\prime} \mathbf{\Delta} \mu_{\mathcal{M}}^{\prime}$. Thus, $f$ is welldefined.

We continue to show that the four properties in Definition 9 hold for $f$. Property 1 is ensured since for every $\mathcal{M}, f(\mathcal{M})$ is closed under $\beta$. Indeed, either $f(\mathcal{M})=\operatorname{Mod}\left(\psi_{\mathcal{M}} \boldsymbol{\Delta} \mu_{\mathcal{M}}\right)$ and since $\psi_{\mathcal{M}} \boldsymbol{\Delta} \mu_{\mathcal{M}} \in \mathcal{L}^{\prime}$ its set of models is closed under $\beta$, or $f(\mathcal{M})=C l_{\beta}(\mathcal{M})$. Let us show Property 2 , i.e., $f(\mathcal{M}) \subseteq C l_{\beta}(\mathcal{M})$ for any set of interpretations $\mathcal{M}$. It is obvious when $\mathcal{M}=\varnothing$ (then $f(\mathcal{M})=\varnothing)$, as well as when $f(\mathcal{M})=C l_{\beta}(\mathcal{M})$. Otherwise $f(\mathcal{M})=$ $\operatorname{Mod}\left(\psi_{\mathcal{M}} \boldsymbol{\Delta} \mu_{\mathcal{M}}\right)$ and since $\boldsymbol{\Delta}$ satisfies containment $\operatorname{Mod}\left(\psi_{\mathcal{M}} \boldsymbol{\Delta} \mu_{\mathcal{M}}\right) \subseteq C l_{\beta}\left(\operatorname{Mod}\left(\psi_{\mathcal{M}} \triangle \mu_{\mathcal{M}}\right)\right.$. Therefore in any case we have $f(\mathcal{M}) \subseteq C l_{\beta}(\mathcal{M})$. For showing Property 3 let us consider $\mathcal{M} \neq \varnothing$ such that $\mathcal{M}=C l_{\beta}(\mathcal{M})$. If $f(\mathcal{M})=C l_{\beta}(\mathcal{M})$, then $f(\mathcal{M})=\mathcal{M}$. Otherwise, $f(\mathcal{M})=\operatorname{Mod}\left(\psi_{\mathcal{M}} \Delta \mu_{\mathcal{M}}\right)$ where $\psi_{\mathcal{M}}, \mu_{\mathcal{M}} \in \mathcal{L}^{\prime}$ such that $\operatorname{Mod}\left(\psi_{\mathcal{M}} \triangle \mu_{\mathcal{M}}\right)=\mathcal{M}$. Since satisfies invariance $\operatorname{Mod}\left(\psi_{\mathcal{M}} \boldsymbol{\Delta} \mu_{\mathcal{M}}\right)=\mathcal{M}$. Thus, in any case, $f(\mathcal{M})=\mathcal{M}$. Property 4 is ensured by consistency of $\boldsymbol{\Lambda}$.

Hence, $\beta$-mappings allow us to define refined belief change operators. We give some examples of $\beta$-mappings in the next section (see Section 4.2) and study how they perform to refine update operators.

\section{Update Operators within Fragments}

The previous section presented refinements for any belief change operation. We now focus on refinements for belief update. We recall that a belief update operator is a function $\diamond$ $: \mathcal{L} \times \mathcal{L}$ to $\mathcal{L}$ that maps a formula $\psi$ representing the initial agent's beliefs and a formula $\mu$ encoding a change in her environment to a new formula $\psi \diamond \mu$ representing the updated agent's beliefs.

In this section we first present some update operators that are well-suited for any characterizable fragment (Section 4.1). Then we turn to update operators that require refinements. We first propose some $\beta$-mappings (Section 4.2) and then study the logical properties of the refined operators they define (Section 4.3). Finally we address the question of refining update operators so that they can handle the case where only the formula representing the agent's beliefs is in the fragment (Section 4.4).

\subsection{Dependence-Based Update Operators}

There exists a family of update operators that is well-suited for any characterizable fragment, i.e., that provides a result in the fragment, namely dependence-based update operators (Herzig \& Rifi, 1999).

More formally, a dependence is a function that assigns each atom $a$ a set of atoms $\operatorname{dep}(a)$. This dependence function is extended to formulas by $\operatorname{dep}(\mu)=\bigcup_{a \in \operatorname{Var}(\mu)} d e p(a)$. 
Herzig's update operator (Herzig \& Rifi, 1999) is a dependence-based update operator denoted by $\diamond_{H Z}$ and defined by

$$
\operatorname{Mod}\left(\psi \diamond_{H Z} \mu\right)=\left\{m^{\prime} \in \operatorname{Mod}(\mu) \mid \exists m \in \operatorname{Mod}(\psi): m \Delta m^{\prime} \subseteq \operatorname{dep}(\mu)\right\}
$$

Hegner's (1987) operator, denoted by $\diamond_{H}$, is a special case of Herzig's operator where $\operatorname{dep}(\mu)=\operatorname{Var}(\mu)$, and thus is defined by

$$
\operatorname{Mod}\left(\psi \diamond_{H} \mu\right)=\left\{m^{\prime} \in \operatorname{Mod}(\mu) \mid \exists m \in \operatorname{Mod}(\psi): m \Delta m^{\prime} \subseteq \operatorname{Var}(\mu)\right\}
$$

The following proposition shows that these two update operators are well-suited for any characterizable fragments.

Proposition 12. Let $\mathcal{L}^{\prime}$ be a characterizable fragment of propositional logic. Given two formulas $\psi, \mu \in \mathcal{L}^{\prime}$, then $\psi \diamond_{H Z} \mu \in \mathcal{L}^{\prime}$ (in particular, $\psi \diamond_{H} \mu \in \mathcal{L}^{\prime}$ ).

Proof. Let $\mathcal{L}^{\prime}$ be a $\beta$-fragment, $\psi$ and $\mu$ two formulas of $\mathcal{L}^{\prime}$. Let $n_{1}, \ldots, n_{k} \in \operatorname{Mod}\left(\psi \diamond_{H Z} \mu\right)$. According to the definition of Herzig's operator (Herzig \& Rifi, 1999), there exist models $m_{1}, \ldots, m_{k} \in \operatorname{Mod}(\psi)$, such that for each $i=1, \ldots, k$, we have $n_{i} \Delta m_{i} \subseteq \operatorname{dep}(\mu)$. Consider $\beta\left(n_{1}, \ldots, n_{k}\right) \Delta \beta\left(m_{1}, \ldots, m_{k}\right)$. If $x \notin \operatorname{dep}(\mu)$ then for each $i$, we have $n_{i}(x)=m_{i}(x)$, and thus, $\beta\left(n_{1}(x), \ldots, n_{k}(x)\right)=\beta\left(m_{1}(x), \ldots, m_{k}(x)\right)$. Therefore, $\beta\left(n_{1}, \ldots, n_{k}\right) \Delta \beta\left(m_{1}, \ldots, m_{k}\right) \subseteq$ $\operatorname{dep}(\mu)$. Moreover, we have $\mu \in \mathcal{L}^{\prime}$, thus $\beta\left(n_{1}, \ldots, n_{k}\right) \in \operatorname{Mod}(\mu)$. Similarly, $\psi \in \mathcal{L}^{\prime}$, thus $\beta\left(m_{1}, \ldots, m_{k}\right) \in \operatorname{Mod}(\psi)$. Hence, $\beta\left(n_{1}, \ldots, n_{k}\right) \in \operatorname{Mod}\left(\psi \diamond_{H Z} \mu\right)$. Therefore, $\operatorname{Mod}\left(\psi \diamond_{H Z} \mu\right)$ is closed under $\beta$, hence $\psi \diamond_{H Z} \mu \in \mathcal{L}^{\prime}$.

The following example illustrates the behavior of these dependence-based update operators.

Example 13. Let $\psi=\neg a \wedge \neg b$ and $\mu=a$ be Horn formulas. We have $\operatorname{Mod}(\psi)=\{\varnothing\}$, $\operatorname{Mod}(\mu)=\{\{a\},\{a, b\}\}$ and $\operatorname{Var}(\mu)=\{a\}$. Suppose $\operatorname{dep}(\mu)=\{a, b\}$, we have $\operatorname{Mod}\left(\psi \diamond_{H Z} \mu\right)=$ $\{\{a\},\{a, b\}\}$ and $\operatorname{Mod}\left(\psi \diamond_{H} \mu\right)=\{\{a\}\}$. Therefore, the result of update is also in $\mathcal{L}_{\text {Horn }}$.

All update operators considered in this paper proceed as follows to compute the update of $\psi$ by $\mu$ : A model $m^{\prime}$ of $\mu$ is a model of the updated beliefs if there is a model $m$ of $\psi$ such that the "distance" between $m$ and $m^{\prime}$, measured by their symmetric difference $m \Delta m^{\prime}$, satisfies some property. An important feature of the dependence-based update operators, not shared by Forbus' (1989) and Winslett's operators (1988), is that the property $m \Delta m^{\prime}$ has to satisfy depends on $\mu$, and not on $m$.

We now turn to update operators that are not directly suited for fragments of propositional logic and for which refinements make sense. Thanks to Proposition 11, given $\diamond$ an update operator, the family of all its possible update refinements, $\left[\diamond, \mathcal{L}^{\prime}\right]$, is the set of operators $\diamond^{f_{\beta}}$ where $f_{\beta}$ is a $\beta$-mapping. For this reason we first present different $\beta$-mappings and next study the logical properties of the refined operators they define. 


\subsection{Examples of Refined Belief Update Operators}

We now give some examples of $\beta$-mappings. In the following, let $\triangle: \mathcal{L} \times \mathcal{L} \rightarrow \mathcal{L}$ be a belief change operator, and $\mathcal{L}^{\prime} \subseteq \mathcal{L}$ be a fragment of propositional logic such that $\mathcal{L}^{\prime}$ is a $\beta$-fragment for some $\beta \in \mathcal{B}$.

A natural $\beta$-mapping is the $C l_{\beta}$ function that leads to the definition of a closedbased refined belief change operator denoted by $\triangle^{C l_{\beta}}$ and given by $\operatorname{Mod}\left(\psi \triangle^{C l_{\beta}} \mu\right)=$ $C l_{\beta}(\operatorname{Mod}(\psi \triangle \mu))$.

In the following all refined belief change operators are illustrated in the particular case of belief update operators.

The following examples illustrate the closed-based refinement for several propositional fragments.

Example 14. Let $\psi$ and $\mu$ be Horn formulas such that $\operatorname{Mod}(\psi)=\{\{a, b, c\},\{a, b, c, d, e\}\}$ and $\operatorname{Mod}(\mu)=\{\{b, c\},\{c, d\},\{a, b, d\},\{c\},\{d\},\{b\}, \varnothing\}$ as in Example 6. Such formulas exist since their sets of models are closed under intersection. We have $\operatorname{Mod}\left(\psi \diamond_{F} \mu\right)=$ $\{\{b, c\},\{a, b, d\}\}$ and $\operatorname{Mod}\left(\psi \diamond_{W} \mu\right)=\{\{b, c\},\{c, d\},\{a, b, d\}\}$ that are not closed under intersection. So, neither $\psi \diamond_{F} \mu$ nor $\psi \diamond_{W} \mu$ is in $\mathcal{L}_{\text {Horn }}$. The refined operators $\diamond_{F}^{C l} l_{\wedge}$ and $\diamond_{W}^{C l_{\wedge}}$ are defined as $\operatorname{Mod}\left(\psi \diamond_{F}^{C l_{\wedge}} \mu\right)=C l_{\wedge}\left(\operatorname{Mod}\left(\psi \diamond_{F} \mu\right)\right)=\{\{b, c\},\{a, b, d\},\{b\}\}$ and $\operatorname{Mod}\left(\psi \diamond_{W}^{C l_{\wedge}} \mu\right)=C l_{\wedge}\left(\operatorname{Mod}\left(\psi \diamond_{W} \mu\right)\right)=\{\{b, c\},\{c, d\},\{a, b, d\},\{b\},\{c\},\{d\}, \varnothing\}$.

We give now an example that holds both in the Horn and the Krom fragments.

Example 15. Consider $\psi=a \wedge b \wedge c$ and $\mu=(\neg a \vee \neg b) \wedge(\neg b \vee \neg c) \wedge(\neg a \vee \neg c)$. These two formulas are both Horn and Krom. Their respective set of models $\operatorname{Mod}(\psi)=\{\{a, b, c\}\}$ and $\operatorname{Mod}(\mu)=\{\{a\},\{b\},\{c\}, \varnothing\}$ are closed under intersection and under majority. We have $\operatorname{Mod}\left(\psi \diamond_{F} \mu\right)=\operatorname{Mod}\left(\psi \diamond_{W} \mu\right)=\{\{a\},\{b\},\{c\}\}$, which is closed neither under intersection nor under majority. So $\psi \diamond_{F} \mu$ is neither in $\mathcal{L}_{\text {Horn }}$ nor in $\mathcal{L}_{\text {Krom }}$. The refined operators $\diamond_{F}^{C l_{\wedge}}$ and $\diamond_{W}^{C l}$ are defined as $\operatorname{Mod}\left(\psi \diamond_{F}^{C l \wedge} \mu\right)=\operatorname{Mod}\left(\psi \diamond_{W}^{C l \wedge} \mu\right)=\{\{a\},\{b\},\{c\}, \varnothing\}$. The refined operators $\diamond_{F}^{C l_{m_{3} j_{3}}}$ and $\diamond_{W}^{C l_{m_{a j}}}$ operate similarly.

We now give an example in the affine fragment.

Example 16. Let $\psi$ and $\mu$ be affine formulas such that $\operatorname{Mod}(\psi)=\{\{a, b, c\},\{a, d\}\}$ and $\operatorname{Mod}(\mu)=\{\{a\},\{b, c\},\{a, b\},\{c\}\}$. Such formulas exist since these sets of models are closed under the ternary XOR function. We have $\operatorname{Mod}\left(\psi \diamond_{F} \mu\right)=\operatorname{Mod}\left(\psi \diamond_{W} \mu\right)=$ $\{\{a\},\{a, b\},\{b, c\}\}$, which is not closed under the ternary XOR function. So $\psi \diamond_{F} \mu$ is not in $\mathcal{L}_{\text {affine. }}$ The refined operators $\diamond_{F}^{C l_{\oplus_{3}}}$ and $\diamond_{W}^{C l_{\oplus}}$ for $\mathcal{L}_{\text {affine }}$ are defined as $\operatorname{Mod}\left(\psi \diamond_{F}^{C l_{\oplus 3}} \mu\right)=$ $\operatorname{Mod}\left(\psi \diamond_{W}^{C l_{\oplus}} \mu\right)=\{\{a\},\{a, b\},\{b, c\},\{c\}\}$.

Given a total order over interpretations, another $\beta$-mapping is the $\operatorname{Min}_{\beta}$ function that selects the minimal model in this order when the set of models is not closed.

Definition 17. Let $\beta \in \mathcal{B}$ and $\leqslant$ be a fixed total order on the set $2^{\mathcal{U}}$ of interpretations. We define the function $\operatorname{Min}_{\beta}$ as

$$
\operatorname{Min}_{\beta}(\mathcal{M})= \begin{cases}\mathcal{M} & \text { if } C l_{\beta}(\mathcal{M})=\mathcal{M} \\ \left\{\min _{\leqslant}(\mathcal{M})\right\} & \text { otherwise }\end{cases}
$$


The $\operatorname{Min}_{\beta}$ function allows us to define a min-based refined belief change operator, denoted by $\triangle^{\operatorname{Min}_{\beta}}$ and given by $\operatorname{Mod}\left(\psi \triangle^{\operatorname{Min}_{\beta}} \mu\right)=\operatorname{Min}_{\beta}(\operatorname{Mod}(\psi \triangle \mu))$.

Example 18. Let $\psi, \mu \in \mathcal{L}_{\text {Horn }}$ from Example 14. Recall that $\operatorname{Mod}\left(\psi \diamond_{F} \mu\right)=$ $\{\{b, c\},\{a, b, d\}\}$ and $\operatorname{Mod}\left(\psi \diamond_{W} \mu\right)=\{\{b, c\},\{c, d\},\{a, b, d\}\}$. Consider the following order over interpretations: $\{c, d\}<\{b, c\}<\{a, b, d\}$. We thus have $\operatorname{Mod}\left(\psi \diamond_{F} \operatorname{Min}_{\wedge} \mu\right)=$ $\operatorname{Min}_{\wedge}\left(\operatorname{Mod}\left(\psi \diamond_{F} \mu\right)\right)=\{\{b, c\}\}$ and $\operatorname{Mod}\left(\psi \diamond_{W}^{\operatorname{Min}}{ }^{\prime} \mu\right)=\operatorname{Min}_{\wedge}\left(\operatorname{Mod}\left(\psi \diamond_{W} \mu\right)\right)=\{\{c, d\}\}$.

The two $\beta$-mappings $C l_{\beta}$ and $\operatorname{Min}_{\beta}$ represent two extreme functions, the former selecting the closure of the set of interpretations $\mathcal{M}$, the latter selecting only one interpretation of $\mathcal{M}$.

In between these extremes there is a variety of possible $\beta$-mappings. As an example we define an intermediary function, denoted by $\mathcal{P}$ rox $_{\beta}$, which selects a closed subset of interpretations of $\mathrm{Cl}_{\beta}(\mathcal{M})$ that is the closest to $\mathcal{M}$.

For $\mathcal{M} \neq \varnothing$, let $\mathcal{F}(\mathcal{M})$ be the set of nonempty subsets of $C l_{\beta}(\mathcal{M})$ which are closed under $\beta$. This set is defined more formally as follows.

$$
\mathcal{F}(\mathcal{M})=\left\{\mathcal{N} \mid \varnothing \subset \mathcal{N} \subseteq C l_{\beta}(\mathcal{M}) \text { and } \mathcal{N}=C l_{\beta}(\mathcal{N})\right\}
$$

Let $\mathcal{F}_{p}(\mathcal{M})$ be the set of elements of $\mathcal{F}(\mathcal{M})$ that are the closest to $\mathcal{M}$ (in terms of cardinality of the symmetric difference). This set is defined more formally as follows, for all $\mathcal{M} \neq \varnothing$.

$$
\mathcal{F}_{p}(\mathcal{M})=\left\{\mathcal{N} \in \mathcal{F}(\mathcal{M})\left|\forall \mathcal{N}^{\prime} \in \mathcal{F}(\mathcal{M}),\right| \mathcal{M} \Delta \mathcal{N}|\leqslant| \mathcal{M} \Delta \mathcal{N}^{\prime} \mid\right\}
$$

We assign to any fixed total order over interpretations a lexicographic order over subsets of interpretations, denoted by $\leqslant_{l e x}$. The following example illustrates this assignment.

Example 19. Let $m_{1}, m_{2}$ and $m_{3}$ be models such that $m_{1} \leqslant m_{2} \leqslant m_{3}$. Consider the two sets of models $\mathcal{M}_{1}=\left\{m_{1}, m_{3}\right\}$ and $\mathcal{M}_{2}=\left\{m_{2}\right\}$. These sets are respectively represented by their characteristic vector, 101 and 010 , therefore, $\mathcal{M}_{2} \leqslant$ lex $\mathcal{M}_{1}$.

We now formally define the $\operatorname{Prox}_{\beta}$ function as follows.

Definition 20. Let $\beta \in \mathcal{B}$, let $\leqslant$ be a fixed total preorder over interpretations, $\leqslant$ lex its corresponding lexicographic order over subsets of interpretations and $\mathcal{M} \subseteq 2^{\mathcal{U}}$ a set of interpretations. The function $\mathcal{P r o x}_{\beta}$ is defined as follows:

$$
\operatorname{Prox}_{\beta}(\mathcal{M})= \begin{cases}\mathcal{M} & \text { if } C l_{\beta}(\mathcal{M})=\mathcal{M} \\ C l_{\beta}(\mathcal{M}) & \text { if } C l_{\beta}(\mathcal{M}) \neq \mathcal{M} \text { and } C l_{\beta}(\mathcal{M}) \in \mathcal{F}_{p}(\mathcal{M}) \\ \left\{\min _{\leqslant l e x}\left(\mathcal{F}_{p}(\mathcal{M})\right)\right\} & \text { otherwise }\end{cases}
$$

Indeed, $\mathcal{P} \operatorname{rox}_{\beta}$ is a $\beta$-mapping and the refined belief change operator denoted by $\triangle^{\mathcal{P} \text { rox }_{\beta}}$ is given by $\operatorname{Mod}\left(\psi \triangle^{\mathcal{P} \operatorname{rox}_{\beta}} \mu\right)=\operatorname{Prox}_{\beta}(\operatorname{Mod}(\psi \triangle \mu))$.

Example 21. We come back to Example 14 in the Horn fragment, where $\operatorname{Mod}(\psi)=$ $\{\{a, b, c\},\{a, b, c, d, e\}\}$ and $\operatorname{Mod}(\mu)=\{\{b, c\},\{c, d\},\{a, b, d\},\{c\},\{d\},\{b\}, \varnothing\}$. We consider the following order over interpretations : $\varnothing<\{b\}<\{c\}<\{d\}<\{b, c\}<\{c, d\}<\{a, b, d\}$. 


\begin{tabular}{|c||c|c|c|c|c|c|c|}
\hline & $\varnothing$ & $\{b\}$ & $\{c\}$ & $\{d\}$ & $\{b, c\}$ & $\{c, d\}$ & $\{a, b, d\}$ \\
\hline$\{\{b, c\}\}$ & 0 & 0 & 0 & 0 & 1 & 0 & 0 \\
\hline$\{\{c, d\}$ & 0 & 0 & 0 & 0 & 0 & 1 & 0 \\
\hline$\{\{a, b, d\}\}$ & 0 & 0 & 0 & 0 & 0 & 0 & 1 \\
\hline$\{\{b, c\},\{c, d\},\{c\}\}$ & 0 & 0 & 1 & 0 & 1 & 1 & 0 \\
\hline$\{\{b, c\},\{a, b, d\},\{b\}\}$ & 0 & 1 & 0 & 0 & 1 & 0 & 1 \\
\hline$\{\{c, d\},\{a, b, d\},\{d\}\}$ & 0 & 0 & 0 & 1 & 0 & 1 & 1 \\
\hline
\end{tabular}

Table 3: Lexicographic order on subsets of models

We remind that $\mathcal{M}=\operatorname{Mod}\left(\psi \diamond_{F} \mu\right)=\{\{b, c\},\{a, b, d\}\}$.

There are three subsets of $C l_{\wedge}(\mathcal{M})$ that are $\wedge$-closed and at distance 1 from $\mathcal{M}$, and $C l_{\wedge}(\mathcal{M})$ is one of them. Therefore, $\operatorname{Mod}\left(\psi \diamond_{F}^{\mathcal{P} \operatorname{rox}_{\beta}} \mu\right)=\{\{b, c\},\{a, b, d\},\{b\}\}$.

Now let us consider $\mathcal{M}=\operatorname{Mod}\left(\psi \diamond_{W} \mu\right)=\{\{b, c\},\{c, d\},\{a, b, d\}\}$. Observe that $C l_{\wedge}(\mathcal{M})=\{\{b, c\},\{c, d\},\{a, b, d\},\{b\},\{c\},\{d\}, \varnothing\}$. There is no closed subset of $C l_{\wedge}(\mathcal{M})$ which is at distance 1 from $\mathcal{M}$, and six of them are at distance 2 , therefore $\mathcal{F}_{p}(\mathcal{M})$ is made of these six subsets. Since $C l_{\wedge}(\mathcal{M}) \notin \mathcal{F}_{p}(\mathcal{M})$ we have to determine which of its element is the lexicographically minimal one. For this we focus on Table 3 where we can read the lexicographic order assigned to the different elements of $\mathcal{F}_{p}(\mathcal{M})$.

Hence, $\operatorname{Mod}\left(\psi \diamond_{W}^{\mathcal{P} \operatorname{rox}_{\beta}} \mu\right)=\left\{\mathcal{M}_{3}\right\}=\{\{a, b, d\}\}$.

Observe that in this example the three refinements we have considered give different results, $\operatorname{Mod}\left(\psi \diamond_{W}^{\mathcal{P r o x}_{\beta}} \mu\right) \neq \operatorname{Mod}\left(\psi \diamond_{W}^{C l_{\beta}} \mu\right) \neq \operatorname{Mod}\left(\psi \diamond_{W}^{\operatorname{Min}_{\beta}} \mu\right)$.

\subsection{Logical Properties of Refined Belief Update Operators}

In this section we investigate how our refined update operators behave with respect to satisfaction of the KM postulates. We first show that our update refinements preserve the first four KM postulates.

Proposition 22. Let $\diamond$ be an update operator and $\mathcal{L}^{\prime} \subseteq \mathcal{L}$ a characterizable fragment. For $i=1, \ldots, 4$, if $\diamond$ satisfies postulate $(\mathrm{U} i)$, then so does any refinement of this operator in $\mathcal{L}^{\prime}$, $\diamond \in\left[\diamond, \mathcal{L}^{\prime}\right]$

Proof. Suppose $\mathcal{L}^{\prime}$ is a $\beta$-fragment. Thus we can assume that $\bullet\left[\diamond, \mathcal{L}^{\prime}\right]$ is an operator of the form $\diamond f_{\beta}$ where $f_{\beta}$ is a suitable $\beta$-mapping. Since postulates (U1) and (U4) are exactly the same postulates as (R1) and (R4), and since satisfaction of (U3) follows from satisfaction of (R3), according to Creignou et al. (2014, Prop. 6) we only have to deal with (U2). By definition $\operatorname{Mod}(\psi \diamond \mu)=f_{\beta}(\operatorname{Mod}(\psi \diamond \mu))$. Since $\diamond$ satisfies postulate (U2), if $\psi \models \mu$, then $\psi \diamond \mu \equiv \psi$, i.e., $\operatorname{Mod}(\psi \diamond \mu)=\operatorname{Mod}(\psi)$. Therefore, $f_{\beta}(\operatorname{Mod}(\psi \diamond \mu))=f_{\beta}(\operatorname{Mod}(\psi))$. Since $\psi \in \mathcal{L}^{\prime}, f_{\beta}(\operatorname{Mod}(\psi))=\operatorname{Mod}(\psi)$. Thus, $\psi \mu \equiv \psi$.

A natural question is whether there exist refined update operators that satisfy more postulates. We focus on Forbus' (1989) and Winslett's operators (1988) (that satisfy respectively all KM postulates and the first eight ones) refined by $C l_{\beta}, \operatorname{Min}_{\beta}$ and $\mathcal{P r o x}_{\beta}$ (other update operators as well as other refinements have been studied in Ktari, 2016). We discuss the postulates that are expressible in our fragments, namely (U5), (U6) and (U9). 
In the following, within a characterizable fragment, it is implicit that any $\beta$-mapping we refer to, uses the Boolean function $\beta$ which characterizes the fragment. This means that within $\mathcal{L}_{\text {Horn }}$ (resp. $\mathcal{L}_{\text {Krom }}, \mathcal{L}_{\text {affine }}$ ) a $\beta$-mapping is an $\wedge$-mapping (resp., maj $3_{3}$-mapping, $\oplus_{3}$-mapping).

We first show that Proposition 22 cannot be extended to postulate (U5). Indeed we get the following negative result for (U5).

Proposition 23. Let $\diamond \in\left\{\diamond_{F}, \diamond_{W}\right\}$. The refined update operators $\diamond^{C l_{\beta}}, \diamond^{\operatorname{Min}_{\beta}}$ and $\diamond^{\operatorname{Prox}_{\beta}}$ violate postulate $(\mathrm{U} 5)$ in any $\mathcal{L}^{\prime} \in\left\{\mathcal{L}_{\text {Horn }}, \mathcal{L}_{\text {Krom }}, \mathcal{L}_{\text {affine }}\right\}$.

Proof. The proof is in the appendix.

Observation 1. Let us emphasize that this result shows a difference between revision and update. Indeed, let us recall that Forbus' operator $\diamond_{F}$ (1989) can be considered as the update counterpart of Dalal's (1988) revision operator, ${ }^{\circ} D$. The refinements of these two operators by the function $\operatorname{Min}_{\beta}$ show a different behavior. While Creignou et al. (2014) proved that ${ }^{\circ}{ }_{D}^{\operatorname{Min}_{\beta}}$ satisfies (R5), the above proposition shows that $\diamond_{F}^{\operatorname{Min}_{\beta}}$ violates (U5). Interestingly the proof that $\circ_{D}^{\mathrm{Min}_{\beta}}$ satisfies (R5) relies on the fact that Dalal's operator ${ }^{\circ}$ satisfies both (R5) and (R6). In the context of update (U9) is a weaker version of (R6), that applies only to complete formulas. While Forbus' operator $\diamond_{F}$ satisfies (U9) it can be proved that it does not satisfy (R6) (see the example given in the proof of Proposition 23 for the min refinement in the Horn fragment). This explains the difference of behavior of the two operators, Dalal and Forbus, with respect to the preservation of the fifth postulate, resp. (R5) and (U5).

For the ninth postulate (U9), we obtain a rather general negative result, which is similar to the result obtained for (R6) in the context of revision (Creignou et al., 2014), but which nevertheless requires new examples to be proven, since in the case of update we need complete formulas.

Proposition 24. Let $\diamond \in\left\{\diamond_{F}, \diamond_{W}\right\}$ and $\mathcal{L}^{\prime} \in\left\{\mathcal{L}_{\text {Horn }}, \mathcal{L}_{\text {Krom }}, \mathcal{L}_{\text {affine }}\right\}$. Then any refined operator $\diamond \in\left[\diamond, \mathcal{L}^{\prime}\right]$ violates postulate (U9) in $\mathcal{L}^{\prime}$.

Observe that in order to prove this proposition the examples proposed in Creignou et al. (2014) cannot be used since they do not involve complete formulas and we have to provide new ones.

Proof. The proof is in the appendix.

The status of the sixth postulate (U6) is less clear than the ones we have investigated so far. Indeed, the two following propositions show that the satisfaction of (U6) depends on the $\beta$-mapping that is used to define the refinement.

Proposition 25. Let $\diamond$ be an update operator and $\mathcal{L}^{\prime}$ a $\beta$-fragment. If $\diamond$ satisfies (U6), then so does the refined operator $\diamond \mathrm{Cl}_{\beta}$ in $\mathcal{L}^{\prime}$.

Proof. Suppose that $\left(\psi \diamond C l_{\beta} \mu_{1}\right) \models \mu_{2}$ and $\left(\psi \diamond C l_{\beta} \mu_{2}\right) \models \mu_{1}$. Thus, $C l_{\beta}\left(\operatorname{Mod}\left(\psi \diamond \mu_{1}\right)\right) \subseteq$ $\operatorname{Mod}\left(\mu_{2}\right)$ and $C l_{\beta}\left(\operatorname{Mod}\left(\psi \diamond \mu_{2}\right)\right) \subseteq \operatorname{Mod}\left(\mu_{1}\right)$. Moreover, $\operatorname{Mod}\left(\psi \diamond \mu_{1}\right) \subseteq C l_{\beta}\left(\operatorname{Mod}\left(\psi \diamond \mu_{1}\right)\right)$ and also $\operatorname{Mod}\left(\psi \diamond \mu_{2}\right) \subseteq C l_{\beta}\left(\operatorname{Mod}\left(\psi \diamond \mu_{2}\right)\right)$. Therefore, $\operatorname{Mod}\left(\psi \diamond \mu_{1}\right) \subseteq \operatorname{Mod}\left(\mu_{2}\right)$ and $\operatorname{Mod}\left(\psi \diamond \mu_{2}\right) \subseteq \operatorname{Mod}\left(\mu_{1}\right)$. Since $\diamond$ satisfies (U6), we get $\psi \diamond \mu_{1} \equiv \psi \diamond \mu_{2}$. According to the equivalence property cited in Definition 8, we have finally $\psi \diamond^{C l_{\beta}} \mu_{1} \equiv \psi \diamond^{C l} l_{\beta} \mu_{2}$. 


\begin{tabular}{|c|c|c|c|}
\hline Refined operators & \multicolumn{3}{|c|}{ Postulates } \\
& $(\mathrm{U} 5)$ & $(\mathrm{U} 6)$ & $(\mathrm{U} 9)$ \\
\hline$\diamond_{\boldsymbol{F}}^{C l_{\boldsymbol{\beta}}}$ & $\times$ & $\sqrt{ }$ & $\times$ \\
\hline$\diamond_{\boldsymbol{W}}^{C l_{\boldsymbol{\beta}}}$ & $\times$ & $\sqrt{ }$ & $\times$ \\
\hline$\diamond_{\boldsymbol{F}}^{\boldsymbol{M i n}_{\boldsymbol{\beta}}}$ & $\times$ & $\times$ & $\times$ \\
\hline$\diamond_{\boldsymbol{W}}^{\boldsymbol{M i n}_{\boldsymbol{\beta}}}$ & $\times$ & $\times$ & $\times$ \\
\hline \hline$\diamond_{\boldsymbol{F}}^{\mathcal{P r o x}_{\boldsymbol{\beta}}}$ & $\times$ & $\times{ }_{\mathcal{L}_{\text {Horn }}}$ & $\times$ \\
\hline$\diamond_{\boldsymbol{W}}^{\boldsymbol{P r o x}_{\boldsymbol{\beta}}}$ & $\times$ & $\times_{\mathcal{L}_{\text {Horn }}}$ & $\times$ \\
\hline
\end{tabular}

Table 4: An overview of the satisfied postulates by the refined operators.

Proposition 26. Let $\diamond \in\left\{\diamond_{F}, \diamond_{W}\right\}$. The refined operator $\diamond^{\operatorname{Min}_{\beta}}$ violates postulate (U6) in any $\mathcal{L}^{\prime} \in\left\{\mathcal{L}_{\text {Horn }}, \mathcal{L}_{\text {Krom }}, \mathcal{L}_{\text {affine }}\right\}$.

Proof. The proof is in the appendix.

The refinement by $\mathcal{P r o x}_{\beta}$ of Forbus' (1989) and Winslett's operators (1988) does not seem to behave better than the refinements by $C l_{\beta}$ and $\operatorname{Min}_{\beta}$. It is rather difficult to find counterexamples in all fragments and we obtain only a partial result in $\mathcal{L}_{\text {Horn }}$.

Proposition 27. Let $\diamond \in\left\{\diamond_{F}, \diamond_{W}\right\}$. The refined operator $\diamond^{\mathcal{P r o x}_{\beta}}$ violates postulate (U6) in $\mathcal{L}_{\text {Horn }}$.

Proof. The proof is in the appendix.

Let us briefly summarize and discuss the results obtained in this section so far. Proposition 22 is positive: Given an update operator satisfying the four basic postulates (U1)-(U4), any refinement of it (in any fragment) satisfies them as well. The other results, obtained for refinements of Forbus' (1989) and Winslett's operators (1988), look less promising. Nevertheless they raise interesting issues. On the one hand one might ask whether postulates (U5), (U6) and (U9) should be adapted to refinements, which correspond to a specific way of building update operators. On the other hand one has to bear in mind that Forbus' operator is not the only one satisfying all postulates. Indeed representation theorems (in terms of preorders as discussed in Section 4) characterize operators satisfying all postulates. Some of these operators might lead to refinements satisfying more postulates. A classification of operators that satisfy all postulates and can be refined in such a way to preserve (U5), (U6) and (U9) in some fragment is beyond the scope of this paper and left as future work.

Table 4 gives a general overview of the properties of our refined update operators in terms of satisfaction of the postulates (U5), (U6) and (U9). We put $\sqrt{ }$ if the refined operator satisfies the considered postulate, $\times$ if it violates it in all fragments, and $\times_{\mathcal{L}_{\text {Horn }}}$ it is only known that the refined operator violates the postulate in $\mathcal{L}_{\text {Horn }}$.

Finally observe that (U7) and (U8) are not applicable in our study since they use disjunction of formulas while our fragments are not closed under disjunction (given $\mu_{1}$ and $\mu_{2}$ in $\mathcal{L}^{\prime}, \mu_{1} \vee \mu_{2}$ does not necessarily belong to $\mathcal{L}^{\prime}$ ). These postulates would require to 
be reformulated in order to fit into fragments while still characterizing rational behavior of update operators. This is an interesting issue, which is beyond the scope of this paper. An adapted formulation of these postulates would ideally be validated by a representation theorem.

Let us nevertheless discuss postulate (U8), which is the most uncontroversial postulate for belief update in the context of full propositional logic. It reflects the central fact that a rational update operator should give each model of the original beliefs equal consideration (a property that distinguishes update from revision). Unfortunately (U8) fails playing this role in fragments of propositional logic that are not closed under disjunction. Indeed, the union of closed sets of models obtained after having considered independently each model of the formula representing the belief set, has no reason to be a closed set of models.

However, note that by construction our refined operators first compute the result obtained through an original operator, and then, as a post-processing step, apply a $\beta$-mapping to it. Therefore, starting from an update operator that satisfies (U8) the models of the formula will equally contribute to the update in the first step. So at least the spirit is preserved, even if of course one has to perform a post-processing in order to remain in the fragment. Observe that for the refinement by the closure $C l_{\beta}$, since for for all formulas $\psi$ and $\mu$ in $\mathcal{L}^{\prime}$, $\operatorname{Mod}\left(\psi \diamond C l_{\beta} \mu\right)=C l_{\beta}(\operatorname{Mod}(\psi \diamond \mu))$, we have $T_{\mathcal{L}^{\prime}}\left(\psi \diamond C l_{\beta} \mu\right)=T_{\mathcal{L}^{\prime}}(\psi \diamond \mu)$. Therefore, roughly speaking $\diamond C l_{\beta}$ is the best approximation of $\diamond$ in $\mathcal{L}^{\prime}$, and if $\diamond$ can be considered as a rational update operator, then so can $\diamond C l_{\beta}$ in $\mathcal{L}^{\prime}$.

\subsection{When Only the Formula Representing the Agent's Beliefs is in the Fragment}

When working within fragments a very natural situation is that the formula representing the initial agent's beliefs is indeed in the fragment, while the formula reflecting new information, which potentially comes from an external source, is not. In order to iterate the process one is interested in a result that still belongs to the fragment. An interesting issue is thus to decide whether our approach allows us to refine well-established belief update operators which starting from a formula $\psi$ in the fragment and a formula $\mu$ not necessarily in the fragment, give a result in the fragment. This is what we address in this section (for sake of completeness, the symmetric case, which is much less natural, and in which only new information is required to be in the fragment is addressed in Ktari, 2016).

Given an update operator $\diamond$, we call $\diamond: \mathcal{L}^{\prime} \times \mathcal{L} \rightarrow \mathcal{L}^{\prime}$ a $\diamond$-left-refinement $\left(\right.$ for $\mathcal{L}^{\prime}$ ) if it satisfies all properties given in Definition 8 with $\psi \in \mathcal{L}^{\prime}$ and $\mu \in \mathcal{L}$.

It is then easy to check that the characterization given in Proposition 11 still holds, that is that any $\diamond$-left-refinement can be defined as $\diamond_{\beta}$ for some $\beta$-mapping $f_{\beta}$. So, we are in a position to study the logical properties of such refined operators in terms of satisfaction of postulates.

On the one hand note that the negative results obtained in the initial framework $a$ fortiori hold in this generalized case. On the other hand, the seventh postulate (U7), which did not apply in the previous section, makes sense in this context. For these reasons, we shall examine postulates (U1)-(U4), (U6) and (U7).

We first give a general positive result for three of the four basic postulates. 
Proposition 28. Let $\diamond$ be an update operator and $\mathcal{L}^{\prime} \subseteq \mathcal{L}$ a $\beta$-fragment. For $i=2,3,4$, if $\diamond$ satisfies $(\mathrm{U} i)$, then each $\diamond$-left-refinement for $\mathcal{L}^{\prime}, \boldsymbol{L}^{\prime} \times \mathcal{L} \rightarrow \mathcal{L}^{\prime}$, satisfies postulate $(\mathrm{U} i)$.

Proof. The proof is similar to the one used in Proposition 22, since $\psi \in \mathcal{L}^{\prime}$ is not used for the preservation of (U2)-(U4).

Contrary to (U2), (U3) and (U4), the first postulate (U1) could be violated. The success postulate (U1) says that the models of the updated beliefs have to be models of new information, i.e., $\psi \diamond \mu \models \mu$. In the case of a refined operator $\diamond^{f_{\beta}}, \operatorname{since} \operatorname{Mod}\left(\psi \diamond^{f_{\beta}} \mu\right)=$ $f_{\beta}(\operatorname{Mod}(\mu))$, the problem is that the application of $f_{\beta}$ can generate new models that are not necessarily models of $\mu$, and thus the postulate (U1) is not necessarily preserved. We show that this is indeed the case, and actually we prove that the preservation of (U1) depends on the $\beta$-mapping that is used for the refinement.

Proposition 29. Let $\diamond \in\left\{\diamond_{F}, \diamond_{W}\right\}$ be an update operator and $\mathcal{L}^{\prime} \subseteq \mathcal{L}$ a $\beta$-fragment. The $\diamond$-left-refinement $\diamond l_{\beta}$ violates postulate (U1) in any $\mathcal{L}^{\prime} \in\left\{\mathcal{L}_{\text {Horn }}, \mathcal{L}_{\text {Krom }}, \mathcal{L}_{\text {affine }}\right\}$.

Proof. Let $\diamond \in\left\{\diamond_{F}, \diamond_{W}\right\}$. Consider $\psi \in \mathcal{L}^{\prime}$ such that $\operatorname{Mod}(\psi)=\{\varnothing\}$. This set is closed under $\wedge, \operatorname{maj}_{3}$ and $\oplus_{3}$. Let $\mu \in \mathcal{L}$ such that $\operatorname{Mod}(\mu)=\{\{a\},\{b\},\{c\}\}$, we get $\operatorname{Mod}(\psi \diamond \mu)=$ $\{\{a\},\{b\},\{c\}\}$. Thus, $\operatorname{Mod}\left(\psi \diamond C l_{\beta} \mu\right)=\{\{a\},\{b\},\{c\}, \varnothing\}$. Observe that $\operatorname{Mod}\left(\psi \diamond C l_{\beta} \mu\right) \mp$ $\operatorname{Mod}(\mu)$, hence $\diamond C l_{\beta}$ violates $(\mathrm{U} 1)$.

However, some $\beta$-mappings behave better, in particular the $\beta$-mappings $f$ we call contracting, which are characterized by the property $f(\mathcal{M}) \subseteq \mathcal{M}$ for any set of interpretations $\mathcal{M}$. Observe that $\operatorname{Min}_{\beta}$ is such a contracting mapping.

Proposition 30. Let $\diamond$ be an update operator and $\mathcal{L}^{\prime} \subseteq \mathcal{L}$ be a $\beta$-fragment. If $\diamond$ satisfies (U1) and if $f_{\beta}$ is a contracting $\beta$-mapping, then the $\diamond$-left-refinement, $\diamond^{f_{\beta}}: \mathcal{L}^{\prime} \times \mathcal{L} \rightarrow \mathcal{L}^{\prime}$, satisfies postulate (U1).

Proof. Since $\diamond$ satisfies (U1), we have $\psi \diamond \mu \models \mu$. Thus, $\operatorname{Mod}(\psi \diamond \mu) \subseteq \operatorname{Mod}(\mu)$. Besides, $f_{\beta}$ is contracting, thus $f_{\beta}(\operatorname{Mod}(\psi \diamond \mu)) \subseteq \operatorname{Mod}(\psi \diamond \mu) \subseteq \operatorname{Mod}(\mu)$. Therefore, $\operatorname{Mod}\left(\psi \diamond f_{\beta} \mu\right) \subseteq$ $\operatorname{Mod}(\mu)$, i.e., $\psi \diamond^{f_{\beta}} \mu \models \mu$. Hence $\diamond^{f_{\beta}}$ satisfies (U1).

So interestingly contracting $\beta$-mappings allow us to refine rational update operators in order to obtain update operators defined from $\mathcal{L}^{\prime} \times \mathcal{L}$ to $\mathcal{L}^{\prime}$ that satisfy the four basic postulates. Observe that this is in sharp contrast with belief revision. No refinement of a rational revision operator provides a revision operator defined from $\mathcal{L}^{\prime} \times \mathcal{L}$ to $\mathcal{L}^{\prime}$ that satisfies the first four basic postulates. Indeed, the second postulate for revision (R2) (if $(\psi \wedge \mu)$ is satisfiable then $\psi \circ \mu \equiv \psi \wedge \mu)$ is not compatible with an operator from $\mathcal{L}^{\prime} \times \mathcal{L}$ to $\mathcal{L}^{\prime}$. For instance let us consider $\psi \equiv \top$ and $\mu$ a satisfiable formula which is not equivalent to any formula in $\mathcal{L}^{\prime}$. The formula $\psi \wedge \mu$ is satisfiable since $\psi \wedge \mu \equiv \mu$, whereas $\psi \circ \mu \not \equiv \psi \wedge \mu$ by assumption on the choice of $\mu$.

Another way to deal with (U1) is to consider a weaker version of this postulate that would be more appropriate to fragments in this particular case, where new information does not necessarily belong to the fragment. In full propositional logic (U1) means that $T_{\mathcal{L}}(\mu) \subseteq$ $T_{\mathcal{L}}(\psi \diamond \mu)$. In a fragment $\mathcal{L}^{\prime}$ it would be reasonable to require only that $T_{\mathcal{L}^{\prime}}(\mu) \subseteq T_{\mathcal{L}^{\prime}}(\psi \diamond \mu)$. 
Since for any $\beta$-fragment $\mathcal{L}^{\prime}$ and any formula $\mu, T_{\mathcal{L}^{\prime}}(\mu)=T_{\mathcal{L}^{\prime}}(\tilde{\mu})$ where $\tilde{\mu} \in \mathcal{L}^{\prime}$ is such that $\operatorname{Mod}(\tilde{\mu})=C l_{\beta}(\operatorname{Mod}(\mu))$, we propose the following weaker version of (U1):

Let $\mathcal{L}^{\prime}$ be a $\beta$-fragment, $\psi \in \mathcal{L}^{\prime}$, and $\mu \in \mathcal{L}$.

(Ũ1) $\psi \diamond \mu \models \tilde{\mu}$, where $\tilde{\mu} \in \mathcal{L}^{\prime}$ is $\operatorname{such}$ that $\operatorname{Mod}(\tilde{\mu})=C l_{\beta}(\operatorname{Mod}(\mu))$.

Interestingly, with this more adequate formulation the success postulate is preserved by left-refinements.

Proposition 31. Let $\diamond$ be an update operator and $\mathcal{L}^{\prime} \subseteq \mathcal{L}$ a $\beta$-fragment. If $\diamond$ satisfies (U1), then each $\diamond$-left-refinement for $\mathcal{L}^{\prime}, \diamond: \mathcal{L}^{\prime} \times \mathcal{L} \rightarrow \mathcal{L}^{\prime}$, satisfies postulate (U一).

Proof. Since $\diamond$ satisfies (U1), we have $\psi \diamond \mu \models \mu$. Thus, $\operatorname{Mod}(\psi \diamond \mu) \subseteq \operatorname{Mod}(\mu)$. According to Definition 9 , for any $\beta$-mapping $f_{\beta}$ we have $f_{\beta}(\operatorname{Mod}(\psi \diamond \mu)) \subseteq C l_{\beta}(\operatorname{Mod}(\psi \diamond \mu))$, and

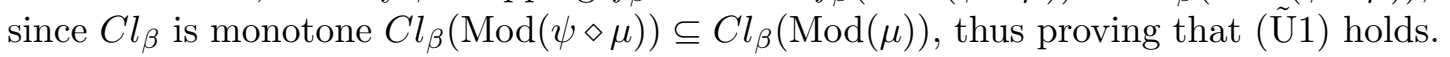

The status of postulate (U6) seems to be unchanged in this generalized framework compared to the original one.

Proposition 32. Let $\diamond$ be an update operator and $\mathcal{L}^{\prime} \subseteq \mathcal{L}$ be a $\beta$-fragment. If $\diamond$ satisfies (U6), then the $\diamond$-left-refinement, $\diamond^{C l_{\beta}}: \mathcal{L}^{\prime} \times \mathcal{L} \rightarrow \mathcal{L}^{\prime}$, satisfies postulate (U6).

Proof. The proof is similar to the one used in Proposition 25, since $\mu \in \mathcal{L}^{\prime}$ is not used.

In the previous section, the seventh postulate (U7) was not applicable since the considered fragments are not closed under disjunction, however for $\diamond$-left-refinements, there is no constraint on new information $\mu$ anymore and this postulate makes sense. We get a negative result for this postulate.

Proposition 33. Let $\diamond \in\left\{\diamond_{F}, \diamond_{W}\right\}$. The $\diamond$-left-refinement $\diamond^{\operatorname{Min}_{\beta}}$ violates postulate (U7) in $\mathcal{L}_{\text {Horn }}$.

Proof. Let $\psi$ be a formula in $\mathcal{L}_{\text {Horn }}$ such that $\operatorname{Mod}(\psi)=\{\{b, c, d\}\}$ and let $\mu_{1}$ and $\mu_{2}$ be two formulas in $\mathcal{L}$ such that $\operatorname{Mod}\left(\mu_{1}\right)=\{\{a, b, c\},\{c\}\}$ and $\operatorname{Mod}\left(\mu_{2}\right)=\{\{a, b, c\},\{d\}\}$. Observe that $\operatorname{Mod}\left(\mu_{1}\right) \cup \operatorname{Mod}\left(\mu_{2}\right)$ is not closed under $\wedge$ and thus $\mu_{1} \vee \mu_{2}$ is not equivalent to any formula in $\mathcal{L}_{\text {Horn }}$. Consider the following order: $\{c\}<\{a, b, c\}<\{d\}$. We get $\operatorname{Mod}(\psi \diamond$ $\left.\mu_{1}\right)=\{\{a, b, c\},\{c\}\}$, which is closed under $\wedge$, thus $\operatorname{Mod}\left(\psi \diamond \operatorname{Min}_{\wedge} \mu_{1}\right)=\{\{a, b, c\},\{c\}\}$. Moreover we have $\operatorname{Mod}\left(\psi \diamond \mu_{2}\right)=\{\{a, b, c\},\{d\}\}$ which is not closed under $\wedge$, this leads to $\operatorname{Mod}\left(\psi \diamond^{\operatorname{Min} \wedge} \mu_{2}\right)=\{\{a, b, c\}\}$. Therefore $\operatorname{Mod}\left(\psi \diamond^{\operatorname{Min} \wedge} \mu_{1}\right) \cap \operatorname{Mod}\left(\psi \diamond^{\operatorname{Min}_{\wedge}} \mu_{2}\right)=\{\{a, b, c\}\}$. Besides, we get $\operatorname{Mod}\left(\psi \diamond\left(\mu_{1} \vee \mu_{2}\right)\right)=\{\{a, b, c\},\{c\},\{d\}\}$, which is not closed under $\wedge$ thus $\operatorname{Mod}\left(\psi \diamond^{\operatorname{Min}_{\wedge}}\left(\mu_{1} \vee \mu_{2}\right)\right)=\{\{c\}\}$. Consequently we have $\operatorname{Mod}\left(\psi \diamond^{\operatorname{Min}_{\wedge}} \mu_{1}\right) \cap \operatorname{Mod}\left(\psi \diamond^{\operatorname{Min}_{\wedge}} \mu_{2}\right) \nsubseteq$ $\operatorname{Mod}\left(\psi \diamond^{\operatorname{Min}_{\wedge}}\left(\mu_{1} \vee \mu_{2}\right)\right)$. Hence, $\diamond^{\operatorname{Min}_{\beta}}$ violates $(U 7)$ in $\mathcal{L}_{\text {Horn }}$.

To conclude this section let us recall that Herzig's update operators $\diamond_{H Z}$ (Herzig \& Rifi, 1999), and in particular Hegner's (1987) operator $\diamond_{H}$, are well-suited for update in any characterizable fragment when both the formula representing the agent's beliefs and the formula reflecting new information are in the fragment (see Proposition12). However, this is not the case anymore when new information is not required to be in the fragment, as illustrated in the following example. 
Example 34. Let two formulas $\psi$ and $\mu$ such that $\psi=\neg a \wedge \neg b \wedge c$ and $\mu=a \vee b$. We have $\operatorname{Mod}(\psi)=\{\{c\}\}, \operatorname{Mod}(\mu)=\{\{a\},\{b\},\{a, b\},\{a, c\},\{b, c\},\{a, b, c\}\}$ and $\operatorname{Var}(\mu)=\{a, b\}$. Clearly, $\psi$ is in $\mathcal{L}_{\text {Horn }}$ but $\mu$ is not equivalent to any Horn formula. Assume dep $(\mu)=\{a, b\}$, we get $\operatorname{Mod}\left(\psi \diamond_{H Z} \mu\right)=\{\{a, c\},\{b, c\},\{a, b, c\}\}$, which is not closed under intersection. Therefore, within this more general framework ( $\diamond$-left-refinement), Herzig's update operators $\diamond_{H Z}$ (Herzig 85 Rifi, 1999), and in particular Hegner's (1987) operator $\diamond_{H}$, would deserve to be refined.

\section{Conclusion}

We have investigated to which extent well-established model-based belief change operators can be refined to work within propositional fragments. We have first defined desired properties any refined belief change operator should satisfy and provided a characterization of all such refined operators. Then, we focused on the belief update operation, which has been neglected so far. We showed that any refinement of an update operator preserves the basic KM update postulates (U1) - (U4) (Katsuno \& Mendelzon, 1992) for any fragment. We then focused on Forbus' (1989) and Winslett's update operators (1988) within Horn, Krom and affine fragments.

We showed that all the proposed refinements violate the fifth postulate (U5). This result is very interesting since it highlights a difference between revision and update. An interesting issue is whether this postulate is indeed violated by any refined update operator. Regarding the sixth postulate (U6) the situation is less clear since the refinement by the closure preserves this postulate, while the other studied refinements do not. It would be interesting to characterize the refined operators that preserve it. We also showed that none of the refinements of Forbus' (1989) and Winslett's operators (1988) satisfies the ninth postulate (U9).

We also studied a natural extension, when only the formula representing the agent's beliefs is in the fragment, and not necessarily new information, that is operators from $\mathcal{L}^{\prime} \times \mathcal{L}$ to $\mathcal{L}^{\prime}$. Our approach can handle this extension. Using $\beta$-mappings that are contracting allows us to define refined update operators, which - contrary to revision - satisfy the first four basic postulates.

There are several interesting issues for future work. The first one concerns the postulates. The KM postulates (Katsuno \& Mendelzon, 1992) that are meaningful in propositional fragment, namely (U5), (U6) and (U9), are not all satisfied by refined operators. An interesting issue is how to weaken them in such a way that some refinements, notably the closure-based refinement, satisfy them. Other KM postulates are not expressible in fragments, namely (U7) and (U8). For them, an additional difficulty is to modify them so that they are expressible in fragments, regardless of refinement operators. A challenging task would be to find an appropriate formulation of these postulates that leads to a representation theorem for update in fragments, as it was already done for revision (Delgrande \& Peppas, 2015) and merging (Haret, Rümmele, \& Woltran, 2017) within the Horn fragment.

Besides, we plan to continue our study in exploring other belief change operations, such as belief erasure and belief forget, which are defined by means of update operators. Finally, an ambitious issue is the study of the computational complexity of the refined update operators. 


\section{Acknowledgments}

This work received support from the french Agence Nationale de la Recherche, ASPIQ project reference ANR-12-BS02-0003. The authors thank the anonymous referees of JAIR for their comments, which helped to improve this article.

\section{Appendix A.}

Proposition 35. Let $\diamond \in\left\{\diamond_{F}, \diamond_{W}\right\}$. The refined update operators $\diamond^{C l_{\beta}}, \diamond^{\operatorname{Min}_{\beta}}$ and $\diamond^{\mathcal{P r o x}_{\beta}}$ violate postulate $(\mathrm{U} 5)$ in any $\mathcal{L}^{\prime} \in\left\{\mathcal{L}_{\text {Horn }}, \mathcal{L}_{\text {Krom }}, \mathcal{L}_{\text {affine }}\right\}$.

Proof. We give first the proof for the refinement by $C l_{\beta}$. For $\mathcal{L}_{\text {Horn }}$ and $\mathcal{L}_{\text {Krom }}$, consider $\psi, \mu, \phi$ in $\mathcal{L}_{\text {Horn }}\left(\right.$ resp. $\left.\mathcal{L}_{\text {Krom }}\right)$ such that $\operatorname{Mod}(\psi)=\{\{a, b, c\}\}, \operatorname{Mod}(\mu)=\{\{a\},\{b\},\{c\}, \varnothing\}$ and $\operatorname{Mod}(\phi)=\{\{c\}, \varnothing\}$. Such formulas exists since these sets of models are closed under $\wedge$ and $\operatorname{maj}_{3}$. For $\diamond \in\left\{\diamond_{F}, \diamond_{W}\right\}$, we have $\operatorname{Mod}(\psi \diamond \mu)=\{\{a\},\{b\},\{c\}\}$ which is not closed under $\wedge$ nor under $\operatorname{maj}_{3}$. We get $\left.\operatorname{Mod}\left(\psi \diamond C l_{\beta} \mu\right)=\{\{a\},\{b\},\{c\}, \varnothing\}\right\}$ and $\operatorname{Mod}\left(\left(\psi \diamond C l_{\beta} \mu\right) \wedge\right.$ $\phi)=\{\{c\}, \varnothing\}\}$. Besides, $\operatorname{Mod}\left(\psi \diamond C l_{\beta}(\mu \wedge \phi)\right)=\{\{c\}\}$, therefore $\operatorname{Mod}\left(\left(\psi \diamond^{C l_{\beta}} \mu\right) \wedge \phi\right) \|$ $\operatorname{Mod}\left(\psi \diamond C l_{\beta}(\mu \wedge \phi)\right)$. Hence, $\diamond^{C l_{\beta}}$ violates $(\mathrm{U} 5)$ in $\mathcal{L}_{\text {Horn }}$ and $\mathcal{L}_{\text {Krom }}$.

For $\mathcal{L}^{\prime}=\mathcal{L}_{\text {affine }}$, consider $\psi, \mu, \phi$ in $\mathcal{L}_{\text {affine }}$ such that $\operatorname{Mod}(\psi)=\{\{a\},\{a, b, c\}\}, \operatorname{Mod}(\mu)=$ $\{\varnothing,\{a, b\},\{a, c\},\{a, d\},\{a, e\},\{b, c\},\{b, d\},\{b, e\},\{c, d\},\{c, e\},\{d, e\},\{a, b, c, d\},\{a, b, c, e\}$, $\{a, b, d, e\},\{a, c, d, e\},\{b, c, d, e\}\}$ and $\operatorname{Mod}(\phi)=\{\{d, e\}, \varnothing\}$. Note that $\psi, \phi \in \mathcal{L}_{\text {affine }}$ since the corresponding sets of models are closed under $\oplus_{3}$ and the set of models of $\mu$ is the set of solutions of the equation $a \oplus b \oplus c \oplus d \oplus e=0$. We have $\operatorname{Mod}(\psi \diamond \mu)=$ $\{\varnothing,\{a, b\},\{a, c\},\{a, d\},\{a, e\},\{b, c\},\{a, b, c, d\},\{a, b, c, e\}\}$. The closure of this set under $\oplus_{3}$ is exactly $\operatorname{Mod}(\mu)$. Hence, $\operatorname{Mod}\left(\psi \diamond C l_{\beta} \mu\right)=\operatorname{Mod}(\mu)$. We now use $\phi \in \mathcal{L}_{\text {affine }}$ with $\operatorname{Mod}(\phi)=$ $\{\{d, e\}, \varnothing\}$. We obtain $\operatorname{Mod}\left(\left(\psi \diamond C l_{\beta} \mu\right) \wedge \phi\right)=\{\varnothing,\{d, e\}\} . \operatorname{But}, \operatorname{Mod}\left(\psi \diamond C l_{\beta}(\mu \wedge \phi)\right)=\{\varnothing\}$. Thus, $\left(\psi \diamond C l_{\beta} \mu\right) \wedge \phi \# \psi \diamond C l_{\beta}(\mu \wedge \phi)$, hence $\diamond_{F}^{C l_{\beta}}$ and $\diamond_{W}^{C l_{\beta}}$ violate postulate $(\mathrm{U} 5)$ in $\mathcal{L}_{\text {affine }}$.

Let us now turn to the refinement by $\operatorname{Min}_{\beta}$. We give first the proof for $\mathcal{L}_{\text {Horn }}$ and $\mathcal{L}_{\text {Krom }}$. Let $\diamond \in\left\{\diamond_{F}, \diamond_{W}\right\}$. Let $\psi, \mu$ and $\phi$ in $\mathcal{L}_{\text {Horn }}$ (resp. $\mathcal{L}_{\text {Krom }}$ ) such that $\operatorname{Mod}(\psi)=\{\{a, b, c, d, e, f\},\{b, c, d, e, f\}\}, \operatorname{Mod}(\mu)=\{\varnothing,\{c\},\{a, b\},\{c, d\},\{e, f\},\{a, b, c\}\}$ and $\operatorname{Mod}(\phi)=\{\{a, b\},\{c, d\},\{e, f\}, \varnothing\}$. Observe that since these sets of models are closed under $\wedge$ (resp. under maj $_{3}$ ) such formulas exist. Consider the following order $\{a, b\}<$ $\{c, d\}<\{e, f\}<\{a, b, c\}$. On the one hand we obtain $\operatorname{Mod}(\psi \diamond \mu)=\{\{c, d\},\{e, f\},\{a, b, c\}\}$, and thus $\operatorname{Mod}\left(\psi \diamond \operatorname{Min}_{\beta} \mu\right)=\{\{c, d\}\}$. Therefore, $\operatorname{Mod}\left(\left(\psi \diamond \operatorname{Min}_{\beta} \mu\right) \wedge \phi\right)=\{\{c, d\}\}$. On the other hand, $\operatorname{Mod}(\psi \diamond(\mu \wedge \phi))=\{\{a, b\},\{c, d\},\{e, f\}\}$, thus $\operatorname{Mod}\left(\psi \diamond \operatorname{Min}_{\beta}(\mu \wedge \phi)\right)=\{\{a, b\}\}$. It is then clear $\left(\psi \diamond^{\operatorname{Min}_{\beta}} \mu\right) \wedge \phi \| \psi \diamond^{\operatorname{Min}_{\beta}}(\mu \wedge \phi)$, hence $\diamond_{F}^{\operatorname{Min}_{\beta}}$ and $\diamond_{W}^{\operatorname{Min}_{\beta}}$ violate postulate (U5) in $\mathcal{L}_{\text {Horn }}$ and $\mathcal{L}_{\text {Krom }}$.

For $\mathcal{L}^{\prime}=\mathcal{L}_{\text {affine }}$, we can consider the formulas $\psi, \mu$ in $\mathcal{L}_{\text {affine }}$ with the same set of models as in the case of the refinement by $C l_{\beta}$ and let $\phi \in \mathcal{L}_{\text {affine }}$ such that $\operatorname{Mod}(\phi)=$ $\{\{b, c\},\{b, d\},\{b, e\},\{b, c, d, e\}\}$. Note that $\phi \in \mathcal{L}_{\text {affine }}$ exists since the corresponding set of models is closed under $\oplus_{3}$. Let us suppose that $\{b, d\}$ and $\{b, c\}$ are the two smallest interpretations with respect to $\leqslant$ with $\{b, d\}<\{b, c\}$. We have on the one hand $\operatorname{Mod}(\psi \diamond \mu)=$ $\{\varnothing,\{a, b\},\{a, c\},\{a, d\},\{a, e\},\{b, c\},\{a, b, c, d\},\{a, b, c, e\}\}$ which is not closed under $\oplus_{3}$ and so $\operatorname{Mod}\left(\psi \diamond^{\operatorname{Min} \beta} \mu\right)=\{\{b, c\}\}$. Hence, $\operatorname{Mod}\left(\left(\psi \diamond^{\operatorname{Min}_{\beta}} \mu\right) \wedge \phi\right)=\{\{b, c\}\}$. On the other hand, we have $\operatorname{Mod}(\psi \diamond(\mu \wedge \phi))=\{\{b, c\},\{b, d\},\{b, e\}\}$, which is not closed under $\oplus_{3}$, and thus 
$\operatorname{Mod}\left(\psi \diamond^{\operatorname{Min}_{\beta}}(\mu \wedge \phi)\right)=\{\{b, d\}\}$. It is obvious that $\left(\psi \diamond^{\operatorname{Min}_{\beta}} \mu\right) \wedge \phi \models \psi \diamond^{\operatorname{Min}_{\beta}}(\mu \wedge \phi)$. Therefore, $\diamond_{F}^{\operatorname{Min}_{\beta}}$ and $\diamond_{W}^{\operatorname{Min}_{\beta}}$ violate postulate (U5) in $\mathcal{L}_{\text {affine }}$.

We now consider the refinement by $\mathcal{P r o x}_{\beta}$. We give first the proof for $\mathcal{L}_{\text {Horn }}$ and $\mathcal{L}_{\text {Krom }}$. Let $\psi, \mu$ and $\phi$ in $\mathcal{L}_{\text {Horn }}\left(\right.$ resp. $\mathcal{L}_{\text {Krom }}$ ) such that $\operatorname{Mod}(\psi)=\{\{a, b, c\}\}$, $\operatorname{Mod}(\mu)=\{\{a\},\{b\},\{c\}, \varnothing\}$ and $\operatorname{Mod}(\phi)=\{\{c\}, \varnothing\}$. For $\diamond \in\left\{\diamond_{F}, \diamond_{W}\right\}$, we have $\operatorname{Mod}(\psi \diamond \mu)=\{\{a\},\{b\},\{c\}\}$, which is not closed under $\wedge\left(\right.$ resp. $\left.\operatorname{maj}_{3}\right)$. Since $\mathcal{F}_{p}(\operatorname{Mod}(\psi \diamond \mu))$ consists in a single set $\{\{a\},\{b\},\{c\}, \varnothing\}$, which is equal to $C l_{\beta}(\operatorname{Mod}(\psi \diamond \mu))$, we have $\operatorname{Mod}\left(\psi \diamond \operatorname{Prox}_{\beta} \mu\right)=\{\{a\},\{b\},\{c\}, \varnothing\}$ and $\operatorname{Mod}\left(\left(\psi \diamond \operatorname{Prox}_{\beta} \mu\right) \wedge \phi\right)=\{\{c\}, \varnothing\}$. On the other hand, $\operatorname{Mod}(\psi \diamond(\mu \wedge \phi))=\{\{c\}\}$, which is closed under $\wedge$ (resp. maj $\left.j_{3}\right)$. Therefore $\operatorname{Mod}\left(\psi \diamond \mathcal{P r o x}_{\beta}(\mu \wedge \phi)\right)=\{\{c\}\}$. It is then clear $\left(\psi \diamond^{\mathcal{P} \operatorname{rox}_{\beta}} \mu\right) \wedge \phi \| \psi \diamond^{\mathcal{P r o x}_{\beta}}(\mu \wedge \phi)$, thus proving that $\diamond_{F}^{\mathcal{P} \operatorname{rox}_{\beta}}$ and $\diamond_{W}^{\mathcal{P}_{W} x_{\beta}}$ violate postulate $(\mathrm{U} 5)$ in $\mathcal{L}_{\text {Horn }}$ and $\mathcal{L}_{\text {Krom }}$.

For $\mathcal{L}^{\prime}=\mathcal{L}_{\text {affine }}$ consider $\psi, \mu$ and $\phi$ in $\mathcal{L}_{\text {affine }}$ such that $\operatorname{Mod}(\psi)=\{\{a, b, c\}\}$, $\operatorname{Mod}(\mu)=\{\varnothing,\{a, b\},\{c, d\},\{c, e\},\{d, e\},\{a, b, c, d\},\{a, b, c, e\},\{a, b, d, e\}\}$ and $\operatorname{Mod}(\phi)=$ $\{\{c, d\},\{c, e\},\{a, b\},\{a, b, d, e\}\}$. Note that such formulas exists in $\mathcal{L}_{\text {affine }}$ since the corresponding sets of models are closed under $\oplus_{3}$. For $\diamond \in\left\{\diamond_{F}, \diamond_{W}\right\}$, we have $\operatorname{Mod}(\psi \diamond \mu)=$ $\{\{a, b\},\{a, b, c, d\},\{a, b, c, e\}\}$ which is not closed under $\oplus_{3}$. On the one hand $\operatorname{Mod}(\psi \diamond \mathcal{P r o x} \oplus$ $\mu)=C l_{\beta}(\{\{a, b\},\{a, b, c, d\},\{a, b, c, e\}\})=\{\{a, b\},\{a, b, c, d\},\{a, b, c, e\},\{a, b, d, e\}\}$, because $C l_{\beta}(\operatorname{Mod}(\psi \diamond \mu))$ is at distance 1 from $\operatorname{Mod}(\psi \diamond \mu)$ and hence in $\mathcal{F}_{p}(\operatorname{Mod}(\psi \diamond \mu))$. Therefore $\operatorname{Mod}((\psi \diamond \mathcal{P} \operatorname{rox} \oplus \mu) \wedge \phi)=\{\{a, b\},\{a, b, d, e\}\}$. On the other hand we have $\operatorname{Mod}(\psi \diamond(\mu \wedge \phi))=$ $\{\{a, b\}\}$. This set of models is closed under $\oplus_{3}$. Thus, $\operatorname{Mod}(\psi \diamond \mathcal{P} \operatorname{Prox} \oplus(\mu \wedge \phi))=\{\{a, b\}\}$. Therefore, $(\psi \diamond \mathcal{P}$ rox $\oplus \mu) \wedge \phi \# \psi \diamond \mathcal{P} \operatorname{rox}_{\oplus}(\mu \wedge \phi)$. Hence, $\diamond_{F}^{\mathcal{P r o x}_{\beta}}$ and $\diamond_{W}^{\mathcal{P} r o x_{\beta}}$ violate postulate (U5) in $\mathcal{L}_{\text {affine }}$.

Proposition 36. Let $\diamond \in\left\{\diamond_{F}, \diamond_{W}\right\}$ and $\mathcal{L}^{\prime} \in\left\{\mathcal{L}_{\text {Horn }}, \mathcal{L}_{\text {Krom }}, \mathcal{L}_{\text {affine }}\right\}$. Then any refined operator $\bullet \in\left[\diamond, \mathcal{L}^{\prime}\right]$ violates postulate (U9) in $\mathcal{L}^{\prime}$.

Proof. First, let us treat the case $\mathcal{L}^{\prime}=\mathcal{L}_{\text {Horn }}$. Consider $\bullet=\diamond^{f}$ where $f$ is a $\wedge$-mapping. Let $\psi$ and $\mu$ in $\mathcal{L}_{\text {Horn }}$ such $\operatorname{Mod}(\psi)=\{\{a, b, c, d\}\}$ and $\operatorname{Mod}(\mu)=$ $\{\{a, b\},\{a, c\},\{a\},\{a, b, e\},\{a, b, c, e\}\}$.

We obtain $\mathcal{M}=\operatorname{Mod}(\psi \diamond \mu)=\{\{a, b\},\{a, c\},\{a, b, c, e\}\}$. Consider the possibilities for $\operatorname{Mod}(\psi \mu)=f(\mathcal{M})$. Recall that $f(\mathcal{M}) \subseteq C l_{\wedge}(\mathcal{M})=\{\{a, b\},\{a, c\},\{a, b, c, e\},\{a\}\}$. We consider two cases:

First assume that $\{a\} \in f(\mathcal{M})$. Let $\phi$ be such that $\operatorname{Mod}(\phi)=\{\{a\},\{a, b, e\}\}=\mathcal{N}$. Clearly, such a $\phi$ exists in $\mathcal{L}_{\text {Horn }}$. Also note that $\operatorname{Mod}(\phi) \subseteq \operatorname{Mod}(\mu)$. We get $\operatorname{Mod}(\psi \wedge \wedge$ $\phi))=\operatorname{Mod}(\psi \phi)=f(\operatorname{Mod}(\psi \diamond \phi))=f(\{\{a\},\{a, b, e\}\})=\mathcal{N}(\mathcal{N}$ is closed under $\wedge, f(\mathcal{N})=$ $\mathcal{N}$ holds by definition of refined operators $)$, but $\operatorname{Mod}((\psi \wedge) \wedge \phi)=f(\mathcal{M}) \cap \operatorname{Mod}(\phi)=\{\{a\}\}$.

Otherwise $\{a\} \notin f(\mathcal{M})$. Since $f(\mathcal{M}) \neq \varnothing$ and $f(\mathcal{M})$ is closed under $\wedge$, by symmetry of the role played by the variables $b$ and $c$, it is sufficient to examine three possibilities for $f(\mathcal{M})$ : either $f(\mathcal{M})=\{\{a, b\}\}$ or $f(\mathcal{M})=\{\{a, b, c, e\}\}$ or $f(\mathcal{M})=\{\{a, b\},\{a, b, c, e\}\}$.

- If $f(\mathcal{M})=\{\{a, b\}\}$ or $f(\mathcal{M})=\{\{a, b, c, e\}\}$, let us consider the formula $\phi$ such that $\operatorname{Mod}(\phi)=\{\{a, b\},\{a, b, c, e\}\}$. Clearly, such a $\phi$ exists in $\mathcal{L}_{\text {Horn }}$. We obtain $\operatorname{Mod}(\psi(\mu \wedge \phi))=\{\{a, b\},\{a, b, c, e\}\}$, whereas $\operatorname{Mod}((\psi \wedge) \wedge \phi)=f(\mathcal{M}) \cap \operatorname{Mod}(\phi)=$ $f(\mathcal{M})$. 
- If $f(\mathcal{M})=\left\{\{a, b\},\{\{a, b, c, e\}\}\right.$. Consider $\phi$ in $\mathcal{L}_{\text {Horn }}$ such that $\operatorname{Mod}(\phi)=$ $\{\{a, c\},\{a, b, c, e\}\} . \quad$ Observe that $\operatorname{Mod}(\psi \wedge(\mu \wedge \phi))=\{\{a, c\},\{a, b, c, e\}\}$, but $\operatorname{Mod}((\psi \mu) \wedge \phi)=f(\mathcal{M}) \cap \operatorname{Mod}(\phi)=\{\{a, b, c, e\}\}$.

Therefore, in any case $\operatorname{Mod}((\psi \wedge) \wedge \phi) \neq \varnothing$ and $\operatorname{Mod}(\psi \wedge(\mu \wedge \phi)) \nsubseteq \operatorname{Mod}((\psi \wedge) \wedge \phi)$, thus proving that $(\psi \wedge \mu) \wedge \phi$ is satisfiable, whereas $\psi \wedge \wedge \phi) \models(\psi \wedge \mu) \wedge \phi$ in $\mathcal{L}_{\text {Horn }}$.

For $\mathcal{L}^{\prime}=\mathcal{L}_{\text {Krom }}$, the formulas $\psi, \mu \in \mathcal{L}_{\text {Krom }}$ with $\operatorname{Mod}(\psi)=\{\{a, b, c, d, e\}\}$, $\operatorname{Mod}(\mu)=\{\{a, b, c\},\{b, c, d\},\{b, c, e\},\{b, c\},\{a, b\}\}$ can be employed. For $\diamond \in\left\{\diamond_{F}, \diamond_{W}\right\}$, we have $\mathcal{M}=\operatorname{Mod}(\psi \diamond \mu)=\{\{a, b, c\},\{b, c, d\},\{b, c, e\}\}$. Observe that $C l_{\text {maj }_{3}}(\mathcal{M})=$ $\{\{a, b, c\},\{b, c, d\},\{b, c, e\},\{b, c\}\}$. Let us consider the possibilities for $\operatorname{Mod}(\psi \mu)=f(\mathcal{M})$. By definition of refined operators, we know that $\{a, b\} \notin f(\mathcal{M})$ since $\{a, b\} \notin C l_{\text {maj }_{3}}(\mathcal{M})$. We consider two cases:

First assume $\{b, c\} \in f(\mathcal{M})$ : Let $\phi$ be such that $\operatorname{Mod}(\phi)=\{\{b, c\},\{a, b\}\}=\mathcal{N}$. Clearly such a $\phi$ exists in $\mathcal{L}_{\text {Krom }}$. Besides note that $\operatorname{Mod}(\phi) \subseteq \operatorname{Mod}(\mu)$. We get $\operatorname{Mod}(\psi \wedge(\mu \wedge \phi))=$ $\operatorname{Mod}(\psi \phi)=f(\operatorname{Mod}(\psi \diamond \phi))=\{\{b, c\},\{a, b\}\}=\mathcal{N}$, whereas $\operatorname{Mod}((\psi \wedge \mu) \wedge \phi)=\{\{b, c\}\}$.

Otherwise, we have $\{b, c\} \notin f(\mathcal{M})$. Since $f(\mathcal{M}) \neq \varnothing$ and $f(\mathcal{M})$ is already closed under maj $_{3}$, by symmetry of the role played by the variables $a, d$ and $e$, it is sufficient to consider two cases for $f(\mathcal{M})$ : either $f(\mathcal{M})=\{\{a, b, c\},\{b, c, d\}\}$ or $f(\mathcal{M})=\{\{a, b, c\}\}$.

- If $f(\mathcal{M})=\{\{a, b, c\},\{b, c, d\}\}$, let us consider the formula $\phi$ such that $\operatorname{Mod}(\phi)=$ $\{\{a, b, c\},\{b, c, e\}\}$. Clearly, such a $\phi$ exists in $\mathcal{L}_{\text {Krom }}$. We obtain thus $\operatorname{Mod}(\psi \wedge \wedge$ $\phi))=\{\{a, b, c\},\{b, c, e\}\}$. Nevertheles, $\operatorname{Mod}((\psi \wedge) \wedge \phi)=f(\mathcal{M}) \cap \operatorname{Mod}(\phi)=\{\{a, b, c\}\}$.

- If $f(\mathcal{M})=\{\{a, b, c\}\}$. We select $\phi$ in $\mathcal{L}_{\text {Krom }}$ with $\operatorname{Mod}(\phi)=\{\{a, b, c\},\{b, c, d\}\}$. Then, $\operatorname{Mod}(\psi \wedge(\mu \wedge \phi))=\{\{a, b, c\},\{b, c, d\}\}$, whereas $\operatorname{Mod}((\psi \wedge \mu) \wedge \phi)=f(\mathcal{M}) \cap \operatorname{Mod}(\phi)=$ $f(\mathcal{M})=\{\{a, b, c\}\}$.

It is then clear that in any case $\operatorname{Mod}((\psi \wedge \mu) \wedge \phi) \neq \varnothing$ and $\operatorname{Mod}(\psi \wedge(\mu \wedge \phi)) \nsubseteq$ $\operatorname{Mod}((\psi \wedge) \wedge \phi)$, thus showing eventually that $(\psi \wedge \mu) \wedge \phi$ is satisfiable, whereas $\psi \wedge(\mu \wedge \phi) \|$ $(\psi \wedge \mu) \wedge \phi$ in $\mathcal{L}_{\text {Krom }}$.

For $\mathcal{L}^{\prime}=\mathcal{L}_{\text {affine }}$, we use formulas $\psi, \mu \in \mathcal{L}_{\text {affine }}$ with $\operatorname{Mod}(\psi)=\{\{a, b, c\}\}$ and $\operatorname{Mod}(\mu)=$ $\{\varnothing,\{a, b\},\{c, d\},\{c, e\},\{d, e\},\{a, b, c, d\},\{a, b, c, e\},\{a, b, d, e\}\}$. Observe that the set of models of $\mu$ is the set of solutions of the following equations system: $(a \oplus b=0, c \oplus d \oplus e=0)$. We have $\mathcal{M}=\operatorname{Mod}(\psi \diamond \mu)=\{\{a, b\},\{a, b, c, d\},\{a, b, c, e\}\}$. For $\diamond \in\left\{\diamond_{F}, \diamond_{W}\right\}$, we have $C l_{\oplus_{3}}(\mathcal{M})=\operatorname{Mod}\left(\psi \diamond C l_{\oplus_{3}} \mu\right)=\{\{a, b\},\{a, b, c, d\},\{a, b, c, e\},\{a, b, d, e\}\}$. Let us consider the possibilities for $\operatorname{Mod}(\psi \mu)=f(\mathcal{M})$. We distiguish two cases.

First, assume $\{a, b, d, e\} \in f(\mathcal{M})$ : let $\phi$ be such that $\operatorname{Mod}(\phi)=\{\{a, b, d, e\},\{a, b\}\}$. Clearly, such a $\phi$ exists in $\mathcal{L}_{\text {affine. }}$. Also note that $\operatorname{Mod}(\phi) \subseteq \operatorname{Mod}(\mu)$. We obtain on the one hand $\operatorname{Mod}(\psi \wedge \wedge \wedge))=\operatorname{Mod}(\psi \diamond \phi)=f(\operatorname{Mod}(\psi \diamond \phi))=\{\{a, b\}\}$ and on the other hand $\operatorname{Mod}((\psi \mu) \wedge \phi)$ contains $\{\{a, b, d, e\}\}$. Otherwise, we have $\{a, b, d, e\} \notin f(\mathcal{M})$. Since $f(\mathcal{M}) \neq \varnothing$ and $f(\mathcal{M})$ is closed under $\oplus_{3}$, by symmetry of the role played by the variables $d$ and $e$, it is sufficient to distinguish four cases for $f(\mathcal{M})$ : either $f(\mathcal{M})=\{\{a, b\}\}$ or $f(\mathcal{M})=\{\{a, b, c, e\}\}$ or $f(\mathcal{M})=\{\{a, b\},\{a, b, c, e\}\}$ or $f(\mathcal{M})=\{\{a, b, c, d\},\{a, b, c, e\}\}:$

- If $f(\mathcal{M})=\{\{a, b\}\}$ or $f(\mathcal{M})=\{\{a, b, c, e\}\}$, we consider the formula $\phi$ such that $\operatorname{Mod}(\phi)=\{\{a, b\},\{a, b, c, e\}\}$. Clearly, such a $\phi$ exists in $\mathcal{L}_{\text {affine }}$. We obtain $\operatorname{Mod}(\psi \wedge \wedge$ $\phi))=\{\{a, b\},\{a, b, c, e\}\}$, whereas $\operatorname{Mod}((\psi \wedge \mu) \wedge \phi)=f(\mathcal{M}) \cap \operatorname{Mod}(\phi)=f(\mathcal{M})$. 
- If $f(\mathcal{M})=\{\{a, b\},\{a, b, c, e\}\}$. In this case, let consider $\phi$ in $\mathcal{L}_{\text {affine }}$ such that $\operatorname{Mod}(\phi)=$ $\{\{a, b\},\{a, b, c, d\}\}\}$. Observe that while $\operatorname{Mod}(\psi \wedge(\mu \wedge \phi))=\{\{a, b\},\{a, b, c, d\}\}$, $\operatorname{Mod}((\psi \wedge) \wedge \phi)=f(\mathcal{M}) \cap \operatorname{Mod}(\phi)=\{\{a, b\}\}$.

- If $f(\mathcal{M})=\{\{a, b, c, d\},\{a, b, c, e\}\}$, we use $\phi \in \mathcal{L}_{\text {affine }}$ with $\operatorname{Mod}(\phi)=$ $\{\{a, b\},\{a, b, c, d\}\}$. While $\operatorname{Mod}(\psi \wedge(\mu \wedge \phi))=\{\{a, b\},\{a, b, c, d\}\}$, we get $\operatorname{Mod}((\psi \wedge \mu) \wedge$ $\phi)=f(\mathcal{M}) \cap \operatorname{Mod}(\phi)=\{\{a, b, c, d\}\}$.

Obviously, $\operatorname{Mod}((\psi \wedge \mu) \wedge \phi) \neq \varnothing$ and $\operatorname{Mod}(\psi \wedge(\mu \wedge \phi)) \nsubseteq \operatorname{Mod}((\psi \wedge \mu) \wedge \phi)$ in all cases, thus proving that $(\psi \wedge) \wedge \phi$ is satisfiable, whereass $\psi \wedge \wedge \phi) \models(\psi \wedge \mu) \wedge \phi$ in $\mathcal{L}_{\text {affine }}$.

Proposition 37. Let $\diamond \in\left\{\diamond_{F}, \diamond_{W}\right\}$. The refined operator $\diamond^{\operatorname{Min}_{\beta}}$ violates postulate (U6) in any $\mathcal{L}^{\prime} \in\left\{\mathcal{L}_{\text {Horn }}, \mathcal{L}_{\text {Krom }}, \mathcal{L}_{\text {affine }}\right\}$.

Proof. Let $\diamond \in\left\{\diamond_{F}, \diamond_{W}\right\}$. We give first the proof for $\mathcal{L}_{\text {Horn }}$. Let $\psi, \mu_{1}, \mu_{2} \in \mathcal{L}_{\text {Horn }}$ with $\operatorname{Mod}(\psi)=\{\{b\},\{a, b, c, d\}\}, \operatorname{Mod}\left(\mu_{1}\right)=\{\{a\},\{a, b\},\{a, c\},\{a, b, c, e\}\}$ and $\operatorname{Mod}\left(\mu_{2}\right)=\{\{a, b\}$, $\{a, b, c, e\}\}$. Suppose that $\{a, b\}<\{a, c\}<\{a, b, c, e\}$. On the one hand, we have $\operatorname{Mod}(\psi \diamond$ $\left.\mu_{1}\right)=\{\{a, b\},\{a, c\},\{a, b, c, e\}\}$ which is not closed under $\wedge$. Thus, $\operatorname{Mod}\left(\psi \diamond^{\operatorname{Min}_{\wedge}} \mu_{1}\right)=$ $\operatorname{Min}_{\wedge}(\{\{a, b\},\{a, c\},\{a, b, c, e\}\})=\{\{a, b\}\} \subseteq \operatorname{Mod}\left(\mu_{2}\right)$. On the other hand, we have $\operatorname{Mod}(\psi \diamond$ $\left.\mu_{2}\right)=\{\{a, b\},\{a, b, c, e\}\}$, a set of models closed under $\wedge$. Therefore, $\operatorname{Mod}\left(\psi \diamond^{\operatorname{Min}_{\wedge}} \mu_{2}\right)=$ $\{\{a, b\},\{a, b, c, e\}\} \subseteq \operatorname{Mod}\left(\mu_{1}\right)$. But, $\psi \diamond^{\operatorname{Min}_{\wedge}} \mu_{1} \not \equiv \psi \diamond^{\operatorname{Min}_{\wedge}} \mu_{2}$, hence $\diamond^{\operatorname{Min}_{\beta}}$ violates (U6) in $\mathcal{L}_{\text {Horn }}$.

For $\mathcal{L}^{\prime}=\mathcal{L}_{\text {Krom }}$, we use $\psi, \mu_{1}, \mu_{2} \in \mathcal{L}_{\text {Krom }}$ with $\operatorname{Mod}(\psi)=\{\{a, b, c\},\{a, b\}\}, \operatorname{Mod}\left(\mu_{1}\right)=$ $\{\{a\},\{b\},\{c\}, \varnothing\}$ and $\operatorname{Mod}\left(\mu_{2}\right)=\{\{a\},\{c\}\}$ and we suppose that $\{a\}<\{b\}<\{c\}$. We have on the one hand $\operatorname{Mod}\left(\psi \diamond \mu_{1}\right)=\{\{a\},\{b\},\{c\}\}$, this set is not closed maj. Consequently, $\operatorname{Mod}\left(\psi \diamond \operatorname{Min}_{\text {maj }_{3}} \mu_{1}\right)=\operatorname{Min}_{\text {maj }_{3}}(\{\{a\},\{b\},\{c\}\})=\{\{a\}\} \subseteq \operatorname{Mod}\left(\mu_{2}\right)$. On the other hand,

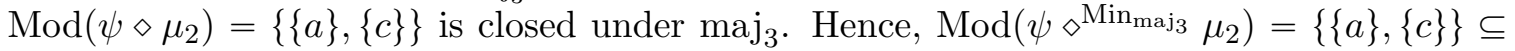
$\operatorname{Mod}\left(\mu_{1}\right)$. Let us notice that $\operatorname{Mod}\left(\psi \diamond^{\operatorname{Min}_{\text {maj }_{3}}} \mu_{1}\right) \neq \operatorname{Mod}\left(\psi \diamond^{\operatorname{Min}_{m_{3}}} \mu_{2}\right)$, hence $\diamond^{\operatorname{Min}_{\beta}}$ violates (U6) in $\mathcal{L}_{\text {Krom }}$.

Finally, for $\mathcal{L}^{\prime}=\mathcal{L}_{\text {affine }}$, the formulas $\psi, \mu_{1}$ and $\mu_{2} \in$ $\mathcal{L}_{\text {affine }}$ with $\operatorname{Mod}(\psi)=\{\{a, b, c, d, e\},\{a, b, c\}\}, \quad \operatorname{Mod}\left(\mu_{1}\right)=$ $\{\varnothing,\{a, b\},\{c, d\},\{e, f\},\{a, b, c, d\},\{a, b, e, f\},\{c, d, e, f\},\{a, b, c, d, e, f\}\}$ and $\operatorname{Mod}\left(\mu_{2}\right)=$ $\{\{a, b\},\{c, d\},\{a, b, e, f\},\{c, d, e, f\}\}$ can be used to show the assertion. Indeed, the set of models of $\mu$ is the set of solutions of the following equation system: $(a \oplus b=0, c \oplus d=0, e \oplus f=0)$. Let us suppose that $\{a, b\}$ is the smallest interpretation with respect to $\leqslant$. On the one hand, we have $\operatorname{Mod}\left(\psi \diamond \mu_{1}\right)=\{\{a, b\},\{a, b, c, d\},\{a, b, c, d, e, f\}\}$, a set not closed under $\oplus_{3}$. We obtain thus $\operatorname{Mod}\left(\psi \diamond^{\min _{\oplus_{3}}} \mu_{1}\right)=\{\{a, b\}\} \subseteq \operatorname{Mod}\left(\mu_{2}\right)$. On the other hand, $\operatorname{Mod}\left(\psi \diamond \mu_{2}\right)=\{\{a, b\},\{c, d\},\{a, b, e, f\},\{c, d, e, f\}\}$ which is already closed under $\oplus_{3}$. Therefore, $\operatorname{Mod}\left(\psi \diamond \min _{\oplus_{3}} \mu_{2}\right)=\{\{a, b\},\{c, d\},\{a, b, e, f\},\{c, d, e, f\}\}$. Hence, $\operatorname{Mod}\left(\psi \diamond^{\min _{\oplus_{3}}} \mu_{2}\right) \subseteq \operatorname{Mod}\left(\mu_{1}\right)$. Nevertheless, $\operatorname{Mod}\left(\psi \diamond^{\min _{\oplus_{3}}} \mu_{1}\right) \neq \operatorname{Mod}\left(\psi \diamond^{\min _{\oplus_{3}}} \mu_{2}\right)$, hence $\diamond \operatorname{Min}_{\beta}$ violates (U6) in $\mathcal{L}_{\text {affine }}$.

Proposition 38. Let $\diamond \in\left\{\diamond_{F}, \diamond_{W}\right\}$. The refined operator $\diamond^{\mathcal{P r o x}_{\beta}}$ violates postulate (U6) in $\mathcal{L}_{\text {Horn }}$. 
Proof. Consider $\psi$ and $\mu_{1}$ two formulas in $\mathcal{L}_{\text {Horn }}$ such that $\operatorname{Mod}(\psi)=\{\{a, b, c, d\}\}$ and $\operatorname{Mod}\left(\mu_{1}\right)=\{\{a, b\},\{a, c\},\{a\},\{a, b, e\},\{a, b, c, e\}\}$. Note that these sets of models are closed under $\wedge$. We get $\operatorname{Mod}\left(\psi \diamond \mu_{1}\right)=\{\{a, b\},\{a, c\},\{a, b, c, e\}\}$, which is not closed by intersection. Observe that $C l_{\beta}\left(\operatorname{Mod}\left(\psi \diamond \mu_{1}\right)\right)$ is at distance 1 from $\operatorname{Mod}\left(\psi \diamond \mu_{1}\right)$, and hence $C l_{\wedge}\left(\operatorname{Mod}\left(\psi \diamond \mu_{1}\right)\right) \in \mathcal{F}_{p}\left(\operatorname{Mod}\left(\psi \diamond \mu_{1}\right)\right)$. Thus, $\operatorname{Mod}\left(\psi \diamond\right.$ Prox $_{\wedge}$ $\left.\mu_{1}\right)=C l_{\wedge}(\{\{a, b\},\{a, c\},\{a, b, c, e\}\})=\{\{a, b\},\{a, c\},\{a, b, c, e\},\{a\}\}$. Let $\mu_{2}$ be a formula in $\mathcal{L}_{\text {Horn }}$ such that $\operatorname{Mod}\left(\mu_{2}\right)=\{\{a, b\},\{a, c\},\{a, b, c, e\},\{a\},\{b, c\},\{b\},\{c\}, \varnothing\}$, we observe that $\operatorname{Mod}\left(\psi \diamond^{\operatorname{Prox}_{\wedge}} \mu_{1}\right) \subseteq \operatorname{Mod}\left(\mu_{2}\right)$. Besides, $\operatorname{Mod}\left(\psi \diamond \mu_{2}\right)=$ $\{\{a, b\},\{a, c\},\{a, b, c, e\},\{b, c\}\}$ is not closed by intersection. We have $\mathcal{F}_{p}\left(\operatorname{Mod}\left(\psi \diamond \mu_{2}\right)\right)=$ $\{\{\{a, b\},\{a, b, c, e\}\},\{\{a, b\},\{a, c\},\{a, b, c, e\},\{a\}\},\{\{a, b\},\{b, c\},\{a, b, c, e\},\{b\}\},\{\{a, c\},\{$ $b, c\},\{a, b, c, e\},\{c\}\}\}$, it does not contain $C l_{\beta}\left(\operatorname{Mod}\left(\psi \diamond \mu_{2}\right)\right)$.

Consider the following order on models of $\mu_{2}$ :

$$
\{a, b\}<\{a, b, c, e\}<\{a\}<\{a, c\}<\{b, c\}<\{b\}<\{c\} .
$$

It induces the following lexicographical order on the sets of models of $\mathcal{F}_{p}(\operatorname{Mod}(\psi \diamond$ $\left.\left.\mu_{2}\right)\right):\{\{a, b\},\{a, b, c, e\}\}<\{\{a, b\},\{a, b, c, e\},\{a\},\{a, c\}\}<\{\{a, b\},\{a, b, c, e\},\{b, c\},\{b\}\}<$ $\{\{a, b, c, e\},\{a, c\}\}<\{\{a, c\},\{b, c\},\{a, b, c, e\},\{c\}\} . \quad \operatorname{Thus}, \operatorname{Mod}\left(\psi \diamond^{\mathcal{P r o x}}{ }_{\wedge} \mu_{2}\right)=$ $\{\{a, b\},\{a, b, c, e\}\} \subseteq \operatorname{Mod}\left(\mu_{1}\right)$. We observe that $\operatorname{Mod}\left(\psi \diamond^{\mathcal{P} r o x_{\wedge}} \mu_{1}\right) \neq \operatorname{Mod}\left(\psi \diamond \mathcal{P r o x}_{\wedge} \mu_{2}\right)$, thus proving that $\diamond^{\mathcal{P} \text { rox }_{\beta}}$ violates $(\mathrm{U} 6)$ in $\mathcal{L}_{\text {Horn }}$.

\section{References}

Alchourrón, C., Gärdenfors, P., \& Makinson, D. (1985). On the logic of theory change: Partial meet contraction and revision functions. Journal of Symbolic Logic, 50, 510530.

Booth, R., Meyer, T., Varzinczak, I., \& Wassermann, R. (2011). On the link between partial meet, kernel, and infra contraction and its application to Horn logic. Journal of Artificial Intelligence Research, 42, 31-53.

Boutilier, C. (1998). A unified model of qualitative belief change: A dynamical systems perspective. Artificial Intelligence, 98(1-2), 281-316.

Cadoli, M., \& Scarcello, F. (2000). Semantical and computational aspects of Horn approximations. Artificial Intelligence, 119(1-2), 1-17.

Creignou, N., Papini, O., Pichler, R., \& Woltran, S. (2014). Belief revision within fragments of propositional logic. Journal of Computer and System Sciences, 80(2), 427-449. A preliminary version appeared in Proc. of KR'2012.

Creignou, N., Papini, O., Rümmele, S., \& Woltran, S. (2016). Belief merging within fragments of propositional logic. ACM Transactions on Computational Logic, 17(3), 20. A preliminary version appeared in Proc. of ECAI'2014.

Creignou, N., Pichler, R., \& Woltran, S. (2017). Do hard SAT-related reasoning tasks become easier in the Krom fragment?. CoRR, abs/1711.07786. 
Creignou, N., \& Vollmer, H. (2008). Boolean constraint satisfaction problems: When does Post's lattice help?. In Complexity of Constraints - An Overview of Current Research Themes [Result of a Dagstuhl Seminar], Vol. 5250 of Lecture Notes in Computer Science, pp. 3-37. Springer.

Dalal, M. (1988). Investigations into theory of knowledge base revision. In Proc. AAAI, pp. 449-479.

del Val, A., \& Shoham, Y. (1994). A unified view of belief revision and update. Journal of Logic and Computation, 4(5), 797-810.

Delgrande, J., Jin, Y., \& Pelletier, F. (2014). Compositional belief update. Journal of Artificial Intelligence Research, 32, 757-791.

Delgrande, J., \& Peppas, P. (2015). Belief revision in Horn theories. Artificial Intelligence, 218, 1-22.

Delgrande, J., \& Wassermann, R. (2013). Horn clause contraction functions. Journal of Artificial Intelligence Research, 48, 457-511.

Doherty, P., Lukaszewicz, W., \& Madalinska-Bugaj, E. (2000). The PMA and relativizing minimal change for action update. Fundamenta Informaticae, 44(1-2), 95-131.

Dubois, D., \& Prade, H. (1993). Belief revision and updates in numerical formalisms: An overview, with new results for the possibilistic framework. In Proc. IJCAI, pp. 620625 .

Eiter, T., \& Gottlob, G. (1992). On the complexity of propositional knowledge base revision, updates, and counterfactuals. Artificial Intelligence, 57(2-3), 227-270.

Fagin, R., Ullman, J., \& Vardi, M. (1983). On the semantic of updates in databases. In Proc. ACM SIGACT SIGMOD, pp. 352-365.

Forbus, K. (1989). Introducing actions into qualitative simulation. In Proc. IJCAI, pp. 1273-1278.

Friedman, N., \& Halpern, J. (1999). Modeling belief in dynamic systems, part II: Revision and update. Journal of Artificial Intelligence Research, 10, 117-167.

Haret, A., Rümmele, S., \& Woltran, S. (2017). Merging in the Horn fragment. ACM Transactions on Computational Logic, 18(1), 6:1-6:32.

Hegner, S. (1987). Specification and implementation of programs for updating incomplete information databases. In Proc. ACM SIGACT-SIGMOD-SIGART, pp. 146-158.

Herzig, A., \& Rifi, O. (1999). Propositional belief base update and minimal change. Artificial Intelligence, 115(1), 107-138.

Horn, A. (1951). On sentences which are true of direct unions of algebras. Journal of Symbolic Logic, 16, 14-21.

Katsuno, H., \& Mendelzon, A. (1991). Propositional knowledge base revision and minimal change. Artificial Intelligence, 52(3), 263-294.

Katsuno, H., \& Mendelzon, A. (1992). On the difference between updating a knowledge base and revising it. In Gärdenfors, P. (Ed.), Belief revision, pp. 183-203. Cambridge University Press. 
Keller, A., \& Winslett, M. (1985). On the use of an extended relational model to handle changing incomplete information. IEEE Transactions of Software Engineering, 11(7), 620-633.

Ktari, R. (2016). Changement de croyances dans des fragments de la logique propositionnelle. Ph.D. thesis, Aix-Marseille Université.

Lang, J. (2007). Belief update revisited. In Proc. IJCAI, pp. 2517-2522.

Liberatore, P., \& Schaerf, M. (2001). Belief revision and update: Complexity of model checking. Journal of Computer and System Sciences, 62(1), 43-72.

Post, E. L. (1941). The two-valued iterative systems of mathematical logic. Annals of Mathematical Studies, 5, 1-122.

Putte, F. V. D. (2013). Prime implicates and relevant belief revision. Journal of Logic and Computation, 23(1), 109-119.

Satoh, K. (1988). Nonmonotonic reasoning by minimal belief revision. In Proc. FGCS, pp. 455-462, Tokyo.

Schaefer, T. J. (1978). The complexity of satisfiability problems. In Proc. STOC, pp. 216-226. ACM Press.

Winslett, M. (1988). Reasoning about action using a possible models approach. In Proc. $A A A I$, pp. 89-93.

Zhang, Y., \& Foo, N. (2000). Updates with disjunctive information: From syntactical and semantical perspectives. Computational Intelligence, 16(1), 29-52.

Zhuang, Z., \& Pagnucco, M. (2014). Entrenchment-based Horn contraction. Journal of Artificial Intelligence Research, 51, 227-254.

Zhuang, Z., Pagnucco, M., \& Zhang, Y. (2013). Definability of Horn revision from Horn contraction. In Proc. IJCAI, pp. 1205-1212. 